Proceedings

\title{
Magnetized Black Holes: Ionized Keplerian Disks and Acceleration of Ultra-High Energy Particles ${ }^{\dagger}$
}

\author{
Zdeněk Stuchlík * (1), Martin Kološ (1) and Arman Tursunov (1) \\ Institute of Physics and Research Centre for Theoretical Physics and Astrophysics, Silesian University in Opava, \\ Bezručovo nám.13, CZ-74601 Opava, Czech Republic; martin.kolos@fpf.slu.cz (M.K.); \\ arman.tursunov@fpf.slu.cz (A.T.) \\ * Correspondence: zdenek.stuchlik@fpf.slu.cz \\ † Presented at the meeting Recent Progress in Relativistic Astrophysics, Shanghai, China, 6-8 May 2019.
}

Published: 24 October 2019

\begin{abstract}
Properties of charged particle motion in the field of magnetized black holes (BHs) imply four possible regimes of behavior of ionized Keplerian disks: survival in regular epicyclic motion, transformation into chaotic toroidal state, destruction due to fall into the $\mathrm{BHs}$, destruction due to escape along magnetic field lines (escape to infinity for disks orbiting Kerr BHs). The regime of the epicyclic motion influenced by very weak magnetic fields can be related to the observed high-frequency quasiperiodic oscillations. In the case of very strong magnetic fields particles escaping to infinity could form UHECR due to extremely efficient magnetic Penrose process - protons with energy $E>10^{21} \mathrm{eV}$ can be accelerated by supermassive black holes with $M \sim 10^{10} \mathrm{M}_{\odot}$ immersed in magnetic field with $B \sim 10^{4} \mathrm{Gs}$.
\end{abstract}

Keywords: black hole; magnetic field; magnetosphere; charged particle dynamic

\section{Introduction}

Magnetic fields surely play very important role in astrophysics-they have been detected and measured in nearly all celestial objects and can have very strong influence on charged matter. Here we focus on the interplay of electromagnetic fields and strong gravity influencing ionized Keplerian disks orbiting magnetized black holes, i.e., black holes immersed in an external magnetic field. We demonstrate that intensity of the magnetic field is crucial for the fate of the ionized Keplerian disks, assumed to be constituted from electrons, protons and ions. The black hole electromagnetic field can have an internal origin, then we have so called Kerr-Newman black hole backgrounds where the motion of charged particles is fully regular [1]. Here we study the case of black holes immersed in an external magnetic field corresponding to generally chaotic motion of charged particles [2-4].

We consider weak magnetic fields satisfying the test field approximation, having thus negligible effect on the background spacetime and on the motion of electrically neutral matter (particles). However, the motion of charged test particles can be influenced by such magnetic fields very strongly. The basic approximation for the weak magnetic fields influencing processes in vicinity of the black hole horizon, frequently used in literature, is the Wald model of the asymptotically uniform magnetic field [5] giving crucial information on the interplay of strong gravity and electromagnetic phenomena $[2-4,6-9]$. On the other hand, for treatment of astrophysical phenomena around magnetic neutron stars the magnetic dipole approximation is considered as basic [10-12].

For a charged test particle with charge $q$ and mass $m$ moving in vicinity of a black hole with mass $M$, surrounded by an external asymptotically uniform magnetic field of the strength $B$, one can introduce a dimensionless "magnetic parameter" $\mathcal{B}$ reflecting the interplay of the electromagnetic 
Lorentz force and the gravity for a given type of charged matter represented by the specific charge $q / m[2,7]$

$$
\mathcal{B}=\frac{B}{2} \frac{q}{m} \frac{G M}{c^{4}}
$$

The magnetic parameter $\mathcal{B}$ can be very large even for weak magnetic fields due to the large value of the specific charge $q / m$, and the influence of the electromagnetic force on the motion of charged particles cannot be neglected even for weak magnetic fields. In our approach the "charged particle" can represent matter ranging from electron, proton, ions, to some massive charged inhomogeneities (charged dust) orbiting in the innermost region of the accretion disk or in its vicinity. The charged particle specific charge $q / m$ then ranges from the electron maximum to zero.

For stellar mass black hole $M \approx 10 \mathrm{M}_{\odot}$, we can have an electron e- in the magnetic field $B \sim 10^{-5} \mathrm{Gs}$, or a charged dust grain (one electron lost, $m=2 \times 10^{-16} \mathrm{~kg}$ ) in the magnetic field $B \sim 10^{9} \mathrm{Gs}$ - and the magnitude of magnetic field parameter is the same in both cases, being $\mathcal{B}=0.004$. Character of the motion is the same in these cases-see also Table 1 for comparison of protons and ions.

Table 1. Intensity of the magnetic field $B$ corresponding to the magnetic parameter $\mathcal{B}=0.1$ for various types of charged particles moving in vicinity of the black hole of mass $M_{B H} \sim 10 \mathrm{M}_{\odot}$.

\begin{tabular}{ccccc}
\hline $\boldsymbol{M}=\mathbf{1 0} \boldsymbol{M}_{\odot} /$ & Electron & Proton & $\mathrm{Fe}+$ & Charged Dust \\
\hline $\mathcal{B}=0.1$ & $10^{-4} \mathrm{Gs}$ & $0.4 \mathrm{Gs}$ & $24 \mathrm{Gs}$ & $10^{10} \mathrm{Gs}$ \\
\hline
\end{tabular}

In the present paper we discuss the fate of ionized Keplerian disks orbiting magnetized black holes. For non-rotating black holes the Keplerian disk can be located in any of the central planes of the Schwarzschild geometry, while around rotating black holes the Keplerian disk has to be located in the equatorial plane of the Kerr geometry due to the so called Bardeen-Peterson effect shifting any inclined disk to the symmetry plane of the Kerr geometry due to the interplay of disk viscosity and geometry frame dragging [13].

We discuss the dependence of the disk fate on the inclination angle to the magnetic field lines, see Figure 1. We start with discussion of the cases of Keplerian disks slightly inclined to the magnetic field lines for three forms of the external magnetic field, namely the uniform, dipole and parabolic; then we concentrate on the behavior of the ionized disk in dependence on its inclination to the magnetic field lines of an uniform magnetic field. We focus on two limiting cases, namely of weak magnetic fields leading to epicyclic motion of the charged particles of the ionized disk, and of strong magnetic fields enabling extremely strong acceleration of ionized matter along the magnetic field lines [14].

We assume that matter of the Keplerian disk follows circular geodesics of the spacetime and it becomes ionized in the internal disk region near the innermost circular geodesics due to irradiation or some other ionization mechanisms, starting to feel the influence of the electromagnetic field (see $[4,7])$. In order to determine the fate of the disk, i.e., motion of the charged particles of the ionized disk, the method of the effective potential seems to be most efficient [8]. Position of the circular charged particle orbits in the combined gravo-electromagnetic field corresponds to the local minima of the effective potential-the crucial role is played by the innermost stable circular orbit (ISCO) representing standard limit on stable circular motion [7]. To have an insight on applicability of the uniform magnetic field approximation, we first compare the dependence of ISCO on the magnetic parameters for the uniform magnetic field, a dipole magnetic field for non-rotating Schwarzschild geometry when we can use in the black hole case the relativistic version of the field generated by a current loop [15]. For completeness we add also the case of parabolic magnetic field as magneto-hydro-dynamic fluid models of thick accretion disks orbiting a black hole demonstrate final creation of a parabolic magnetic field near the rotation axis of the orbiting matter independently of the original configuration of the external magnetic field [16], see Figure 1.

Throughout the present paper we use the spacelike signature $(-,+,+,+)$, and the system of geometric units in which $G=1=c$. 


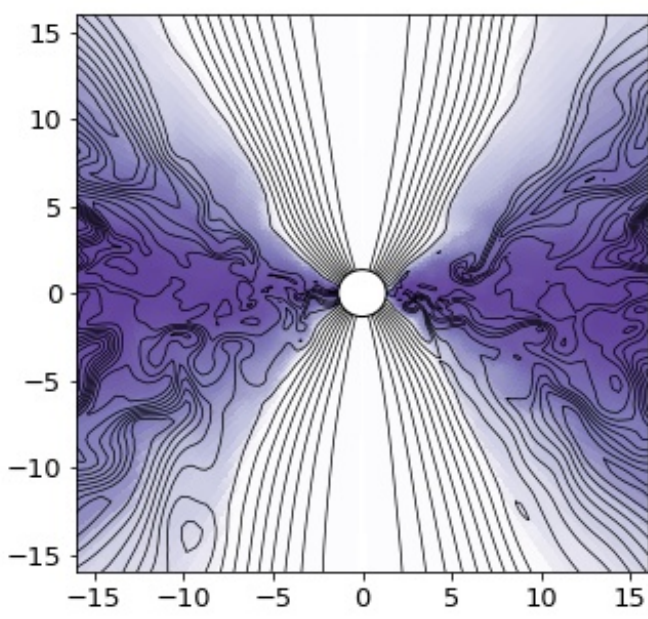

(a)

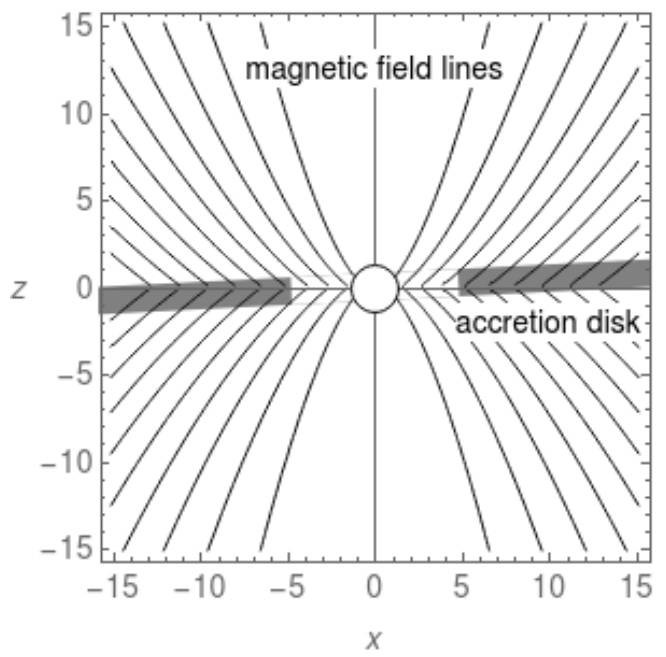

(b)

Figure 1. (a) Magnetic field lines outside a toroidal accretion structure around BH-result of General Relativistic Magnetohydrodynamics simulation in HARM software [17,18]. (b) Schematic picture of a Kerr BH immersed in a parabolic magnetic field and an orbiting Keplerian disk inclined to the field lines.

\section{Ionized Keplerian Disks in Schwarzschild Geometry}

We first study the fate of Keplerian disks around magnetized non-rotating black holes described by the Schwarzschild geometry. Due to this simplification, we can treat in detail the fate of the ionized disk in dependence on the inclination angle between the disk and the magnetic field lines of the asymptotically uniform magnetic field. We first study the dynamics of charged particles in the field of magnetized Schwarzschild black holes assuming three kinds of the external magnetic fields, namely the asymptotically uniform, dipole, and parabolic fields, giving position of the ISCO in dependence on the parameters characterizing the magnetic field. Then we consider the Keplerian disks orbiting a Schwarzschild black hole immersed in a homogeneus magnetic field.

\subsection{Charged Particle Dynamics}

We consider dynamics of a charged particle in the vicinity of the magnetized Schwarzschild black hole using Lorentz equation and related Hamiltonian formalism.

The gravity enters the equations of motion through the vacuum Schwarzschild spacetime line element that takes in the standard Schwarzschild coordinates the form

$$
\mathrm{d} s^{2}=-\left(1-\frac{2 M}{r}\right) \mathrm{d} t^{2}+\left(1-\frac{2 M}{r}\right)^{-1} \mathrm{~d} r^{2}+r^{2}\left(\mathrm{~d} \theta^{2}+\sin ^{2} \theta \mathrm{d} \phi^{2}\right) .
$$

Exact shape of magnetic field around matter accreating black hole is still unknown and there are different approaches to this problem [16,19-21]. Here we test three relevant cases of the external magnetic field around the black hole.

The asymptotically uniform field, having strength $B$ at spatial infinity, assumed to be oriented perpendicularly to the equatorial plane of the black hole spacetime. The nonzero covariant component of the electromagnetic four-vector potential $A^{\mu}$ takes the form [5]

$$
A_{\phi}^{\mathrm{U}}=\frac{B}{2} g_{\phi \phi}
$$

The dipole magnetic field generated by a circular current loop located in the equatorial plane at the radius $a \geq 2$. Outer, $r>a$, solution for the four-vector electromagnetic potential $A^{\mu}$ in the Schwarzschild metric is given by the nonzero covariant component [15] 


$$
A_{\phi}^{\mathrm{D}}=\frac{B}{2}\left[\frac{11}{50}-\ln \left(\frac{5}{4}\right)\right]^{-1}\left[\ln \left(1-\frac{2 M}{r}\right)+\frac{2 M}{r}\left(1+\frac{M}{r}\right)\right] g_{\phi \phi},
$$

where the second term in square brackets is negative for $r>2$.

The parabolic magnetic field predicted by some magnetohydrodynamic simulations is determined by the four-vector electromagnetic potential [16]

$$
A_{\phi}^{\mathrm{P}}=\frac{B}{2} 10^{5 / 4} r^{k}(1-|\cos (\theta)|)
$$

where for declination of the field lines we chose $k=3 / 4$.

All the chosen magnetic fields (uniform, dipole and parabolic) are static, axially symmetry, and have only single nonzero covariant component $A^{\phi}$ of the four-vector electromagnetic potential $A^{\mu}$. The component $A^{\phi}$ has been normalized in such a way that the Lorentz force acting on charged particle will be the same in all three chosen magnetic field configurations at radii $r=10 \mathrm{M}$ in equatorial plane $\theta=\pi / 2$.

Hereafter, we put $M=1$ (if not stated otherwise), i.e., we use dimensionless radial coordinate $r$ (and time coordinate $t$ ). Cartesian coordinates can be found by the coordinate transformations

$$
x=r \cos (\phi) \sin (\theta), \quad y=r \sin (\phi) \sin (\theta), \quad z=r \cos (\theta) .
$$

The equations of motion of a charged particle with charge $q$ and mass $m$ can be expressed using the Hamiltonian formalism

$$
\frac{\mathrm{d} x^{\mu}}{\mathrm{d} \zeta}=\frac{\partial H}{\partial \pi_{\mu}}, \quad \frac{\mathrm{d} \pi_{\mu}}{\mathrm{d} \zeta}=-\frac{\partial H}{\partial x^{\mu}}, \quad H=\frac{1}{2} g^{\alpha \beta}\left(\pi_{\alpha}-q A_{\alpha}\right)\left(\pi_{\beta}-q A_{\beta}\right)+\frac{m^{2}}{2}=0,
$$

where the kinematical four-momentum $p^{\mu}=m u^{\mu}=\mathrm{d} x^{\mu} / \mathrm{d} \zeta$ is related to the generalized (canonical) four-momentum $\pi^{\mu}$ by the relation $\pi^{\mu}=p^{\mu}+q A^{\mu}$. The affine parameter $\zeta$ of the particle is related to its proper time $\tau$ by the relation $\zeta=\tau / m$. The Hamilton Equations (7) represent a set of eight first order ordinary differential equations. The main advantage of the Hamiltonian formalism is the possibility to use very precise numerical integration scheme (symplectic integrator) [22].

Due to the symmetries of the Schwarzschild spacetime (2) and the uniform, dipole, and parabolic magnetic fields (3)-(5), one can easily find the conserved quantities that are particle specific energy and specific axial angular momentum

$$
\mathcal{E}=\frac{E}{m}=-\frac{\pi_{t}}{m}=-g_{t t} u^{t}, \quad \mathcal{L}=\frac{L}{m}=\frac{\pi_{\phi}}{m}=g_{\phi \phi} u^{\phi}+\frac{q}{m} A_{\phi} .
$$

Using these motion constants (motion constants are also the charge and mass of the particle), we can rewrite the Hamiltonian (7) in the form

$$
H=\frac{1}{2} g^{r r} p_{r}^{2}+\frac{1}{2} g^{\theta \theta} p_{\theta}^{2}+\frac{1}{2} g^{t t} E^{2}+\frac{1}{2} g^{\phi \phi}\left(L-q A_{\phi}\right)^{2}+\frac{1}{2} m^{2}=H_{\mathrm{D}}+H_{\mathrm{P}}
$$

where we have separated total Hamiltonian $H$ into dynamical $H_{\mathrm{D}}$ (first two terms) and potential $H_{\mathrm{P}}$ (last three terms) parts.

We define new parameters characterizing the particle, and the magnetic field influence: the particle specific charge $\tilde{q}$, the magnetic field parameter $\mathcal{B}$

$$
\tilde{q}=\frac{q}{m}, \quad \mathcal{B}=\frac{q B}{2 m} .
$$

Energetic boundary of the particle motion (given by the conditions $p_{r}=p_{\theta}=0$ ) is reflected by the Equation (9), giving thus the turning points of the motion in the radial and latitudinal direction,

$$
\mathcal{E}^{2}=V_{\text {eff }}(r, \theta),
$$


where we introduce the effective potential of the charged particle motion, $V_{\mathrm{eff}}(r, \theta)$, by the relation

$$
V_{\text {eff }}(r, \theta) \equiv-g_{t t}\left[g^{\phi \phi}\left(\mathcal{L}-\tilde{q} A_{\phi}\right)^{2}+1\right] .
$$

The effective potential $V_{\text {eff }}(r, \theta)$ combines the influence of the gravity potential (first term), with the influence of the centrifugal force potential given by the specific angular momentum $\mathcal{L}$ and electromagnetic potential energy (terms in square brackets). The effective potential (12) shows clear symmetry $(\mathcal{L}, \mathcal{B}) \leftrightarrow(-\mathcal{L},-\mathcal{B})$, hence from now on we focus on the $\mathcal{L}>0$ case only. The positive angular momentum of a particle, $\mathcal{L}>0$, means that the particle is revolved in the counter-clockwise motion around the black hole in the $x-y$ plane. The typical examples of the behaviour of the effective potential $V_{\text {eff }}(r, \theta)$ in dependence on the energy level are presented in Figures 2-4. In fact, these types of the behaviour of the effective potential are crucial for the fate of the ionized Keplerian disks, as they correspond to the bound, captured, and escaping orbits. Only the separation of the bound orbits to the regular epicyclic orbits and chaotic orbits is not determined by the effective potential.
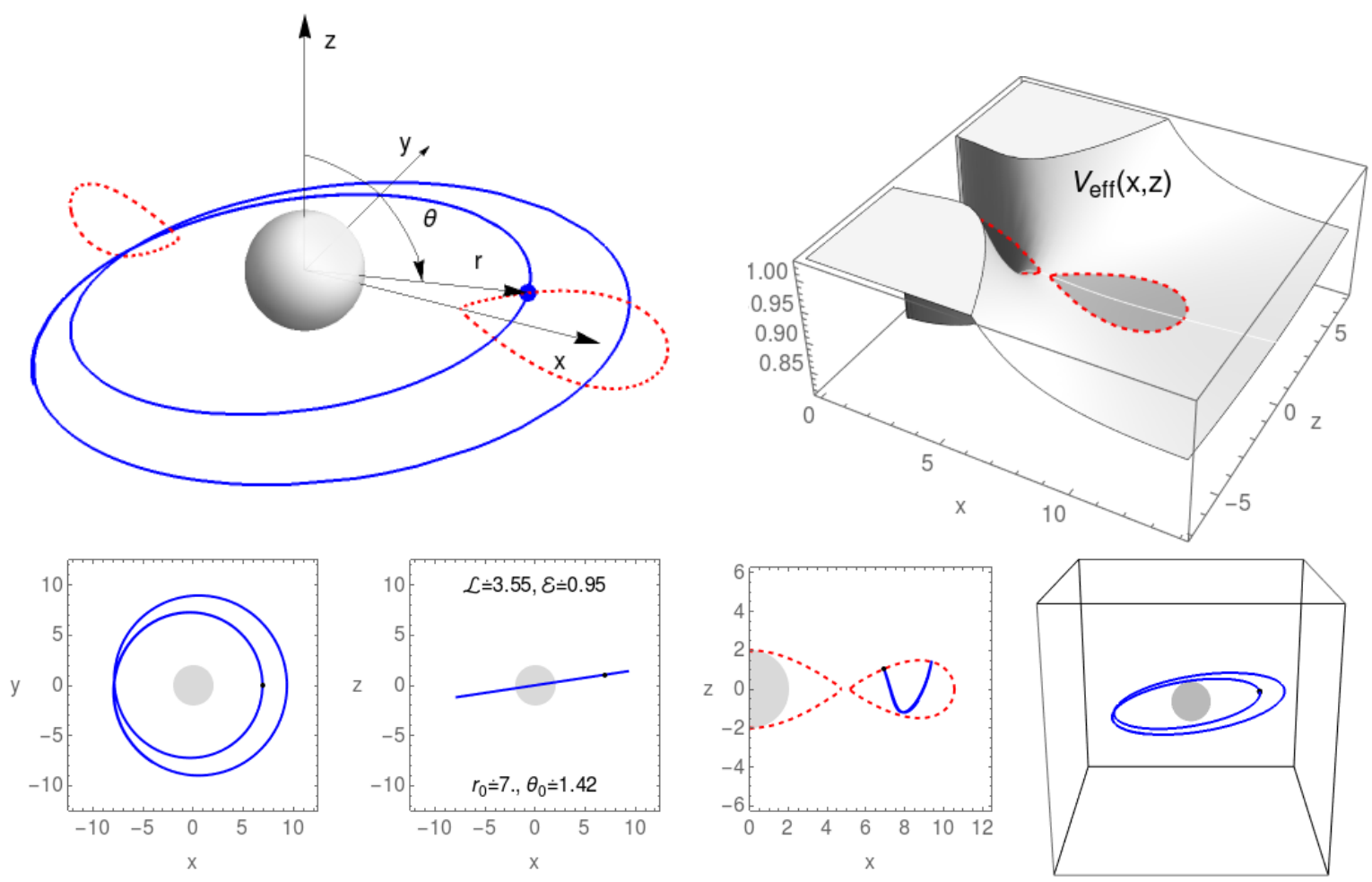

Figure 2. Charged particle on bounded orbit around BH with uniform magnetic filed. Full 3D charged particle trajectory (left figure first row) and representation of its dynamics in effective potential (right figure fist row). Second row of figures show different view on the same particle particle trajectory but from diferent angles.

For the charged particle motion we distinguish from the point of view of the electromagnetic interaction two basic situations

- Lorentz attractive (minus configuration), here with $\mathcal{B}<0$ - magnetic field and angular momentum parameters have opposite signs and the Lorentz force is attracting the charged particle towards the black hole.

$+\quad$ Lorentz repulsive (plus configuration), here with $\mathcal{B}>0$-magnetic field and angular momentum parameters have the same signs and the Lorentz force is repulsive, acting outwards the black hole. 
For the uniform magnetic fields, if charge of the particle is taken positive, $q>0$, the minus configuration $\mathcal{B}<0$ corresponds to the vector of the magnetic field $\vec{B}$ pointing downwards, while plus configuration $\mathcal{B}>0$ corresponds to the vector of the magnetic field $\vec{B}$ pointing upwards the $z$-axis.
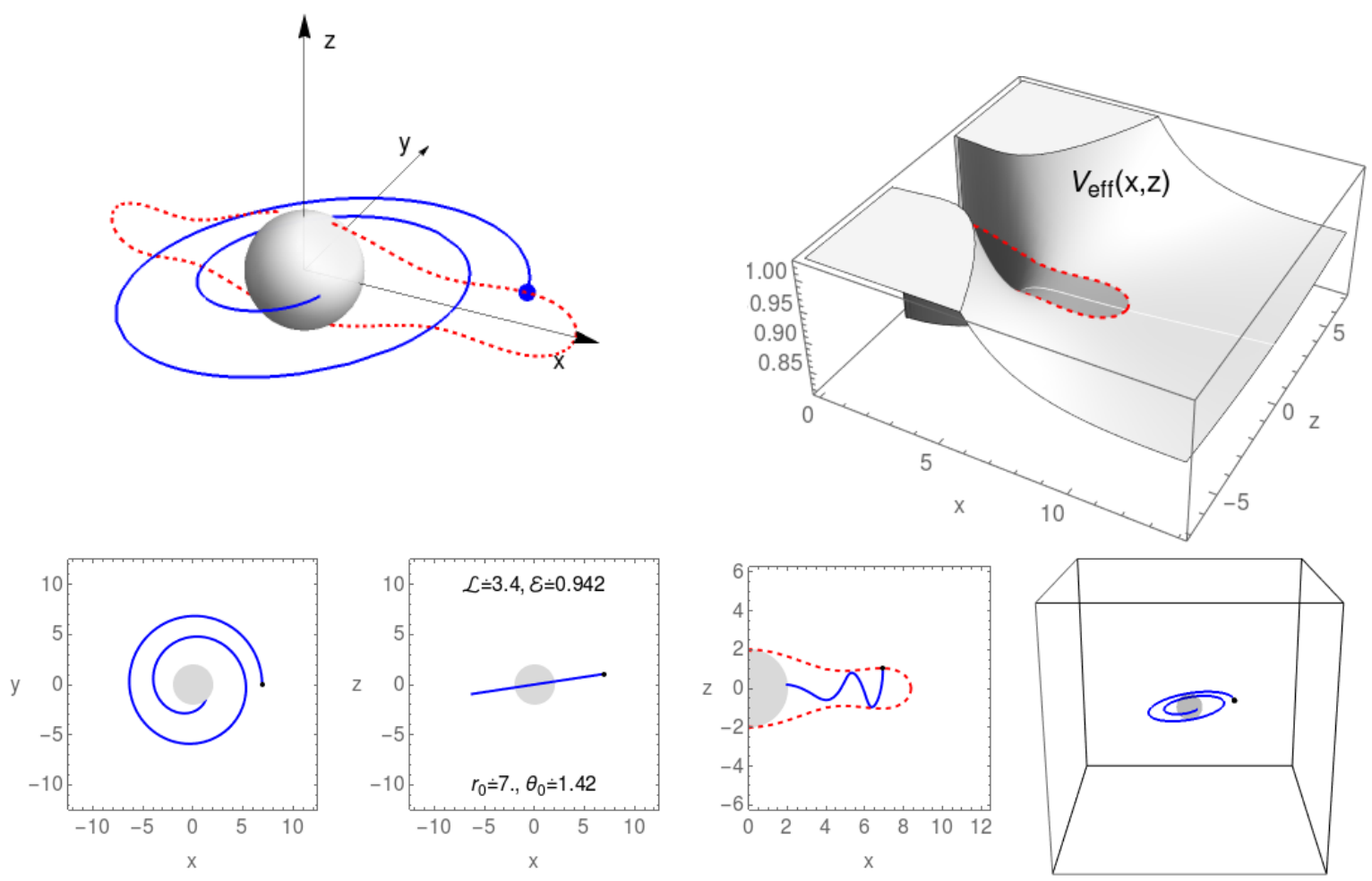

Figure 3. Charged particle captured by BH with magnetic filed, for detailed description see Figure 2.

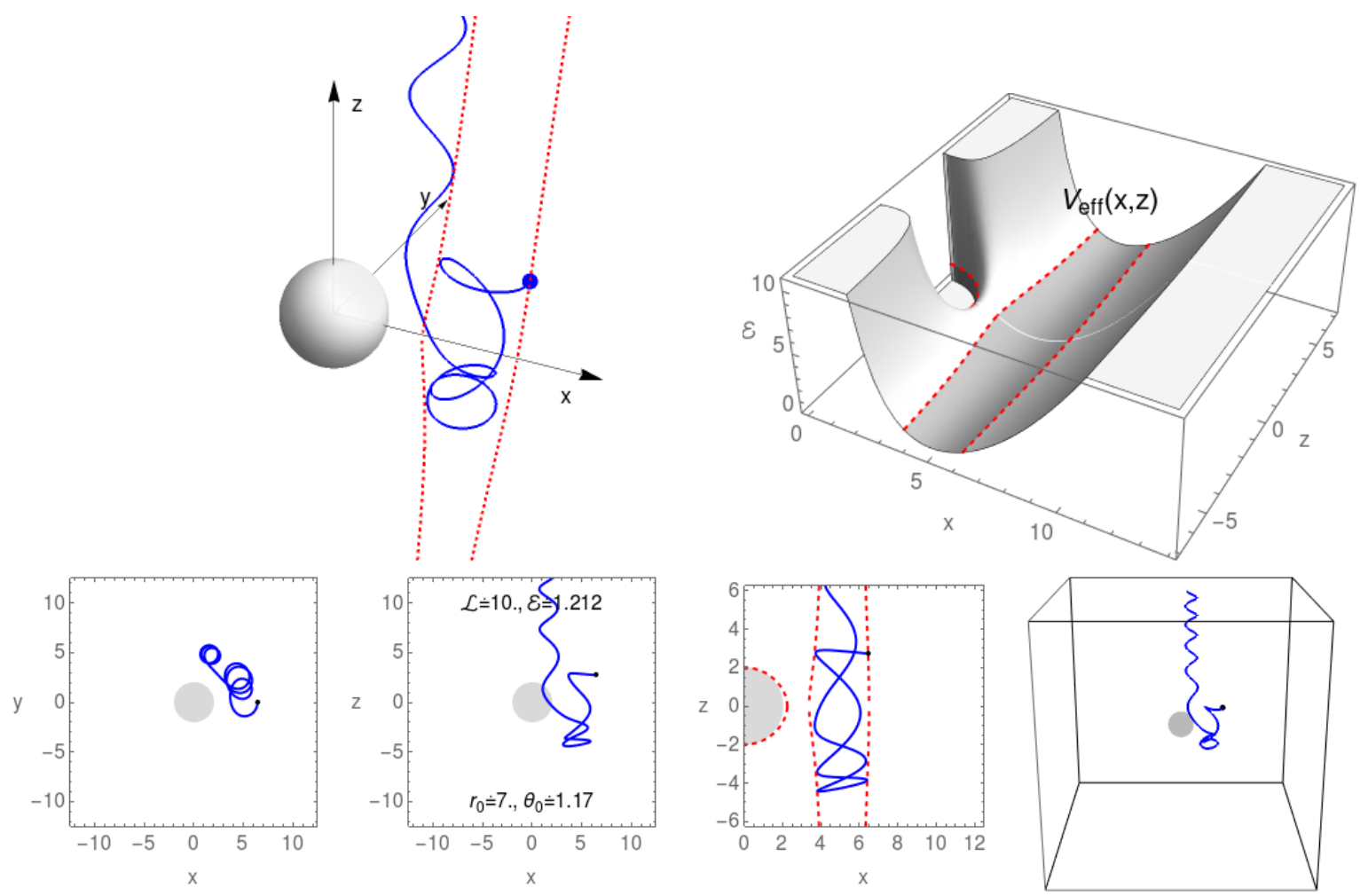

Figure 4. Charged particle escaping from $\mathrm{BH}$ with uniform magnetic filed, for detailed description see Figure 2. 


\subsection{Inner Edge of Stable Circular Orbits}

Electrically neutral particles on circular orbits around black holes (compact objects) can form a Keplerian accretion disk, with its inner edge given by the innermost stable circular geodesic (ISCG) governed only by the spacetime geometry. Similarly, stable circular orbits of charged particles are limited by the innermost stable circular orbit (ISCO) governed by the combined influence of the spacetime geometry and the electromagnetic field. Their position is governed not only by the magnetic field intensity (and the spacetime), but also by the specific charge of the particle. The ISCO is determined by the effective potential $V_{\text {eff }}(r, \theta)$ function.

The stationary points of the effective potential $V_{\text {eff }}(r, \theta)$ are given by $[7,23]$

$$
\partial_{r} V_{\text {eff }}(r, \theta)=0, \quad \partial_{\theta} V_{\text {eff }}(r, \theta)=0
$$

The second equation in the extrema condition (13) has one root at $\theta=\pi / 2$, therefore, the extremum of the $V_{\text {eff }}(r, \theta)$ function is located in the equatorial plane. This extrema can be both stable and unstable. The instability of the circular motion in the latitudinal direction caused by the uniform magnetic field can cause escape of orbiting particles along the magnetic field lines-for details see [4].

The first equation in the extrema condition (13) related to the radius leads to a quadratic equation with respect to the specific angular momentum $\mathcal{L}$

$$
(r-3) \mathcal{L}^{2}+X \mathcal{L}+Y=0
$$

where functions $X, Y$ are given by

$$
X=(r-2) r \tilde{q} \partial_{r} A_{\phi}-2(r-3) \tilde{q} A_{\phi}, \quad Y=(r-3) \tilde{q}^{2} A_{\phi}-(r-2) r \tilde{q}^{2} A_{\phi} \partial_{r} A_{\phi}-r^{2} .
$$

Real roots of radial coordinate $r>2$ from Equation (14) determine maxima, minima and inflex points of the $V_{\text {eff }}(r, \theta=\pi / 2)$ function. Such extrema give stable (minima) and unstable (maxima) equilibrium positions for the circular particle motion, i.e., stable or unstable circular orbits. The inflex points give the marginally stable circular orbits. The solutions of quadratic Equation (14) determine the specific angular momentum $\mathcal{L}_{\mathrm{E} \pm}(r)$ for any circular orbit with radial coordinate $r$

$$
\mathcal{L}_{\mathrm{E} \pm}(r)=\frac{-X \pm \sqrt{X^{2}+4 Y(r-3)}}{2(r-3)} .
$$

Notice that at the radius $r=3=r_{\mathrm{ph}}$ is located the photon circular orbit representing the inner limit for existence of circular geodesics. The local extrema of the $\mathcal{L}_{\mathrm{E} \pm}(r)$ function (16) determine the innermost stable circular orbits (ISCO). The ISCO radial position for charged particle moving around black hole strongly depends on the magnetic field parameter, as demonstrated in Figure 5.

For the uniform magnetic field the charged particle ISCO position is decreasing for both attracting $(\mathcal{B}<0)$ and repulsing $(\mathcal{B}>0)$ Lorentz force and the decrease is significantly stronger for the repulsive force. It is important that for the repulsive force strong enough the ISCO position is located under the photon circular geodesic, but such a situation is impossible for the attractive force. Even for relatively small magnetic field parameter $\mathcal{B}= \pm 0.05$, the ISCO radii are shifted from from $6 M$ to $5 M$ in the geometrized units.

For the dipole (parabolic) magnetic fields, the charged particle ISCO position is shifted outwards from the black hole for attracting Lorentz force $(\mathcal{B}<0)$, while for repulsive Lorentz force $(\mathcal{B}>0)$ the ISCO position is shifted inwards to the black hole. We can see that homogeneity of the magnetic field has a special influence on the position of the ISCO as this radius decreases even for attractive Lorentz forces. Clearly this effect is related to the related modifications of the centrifugal forces governed by the modified axial angular momentum $[7,23]$. 

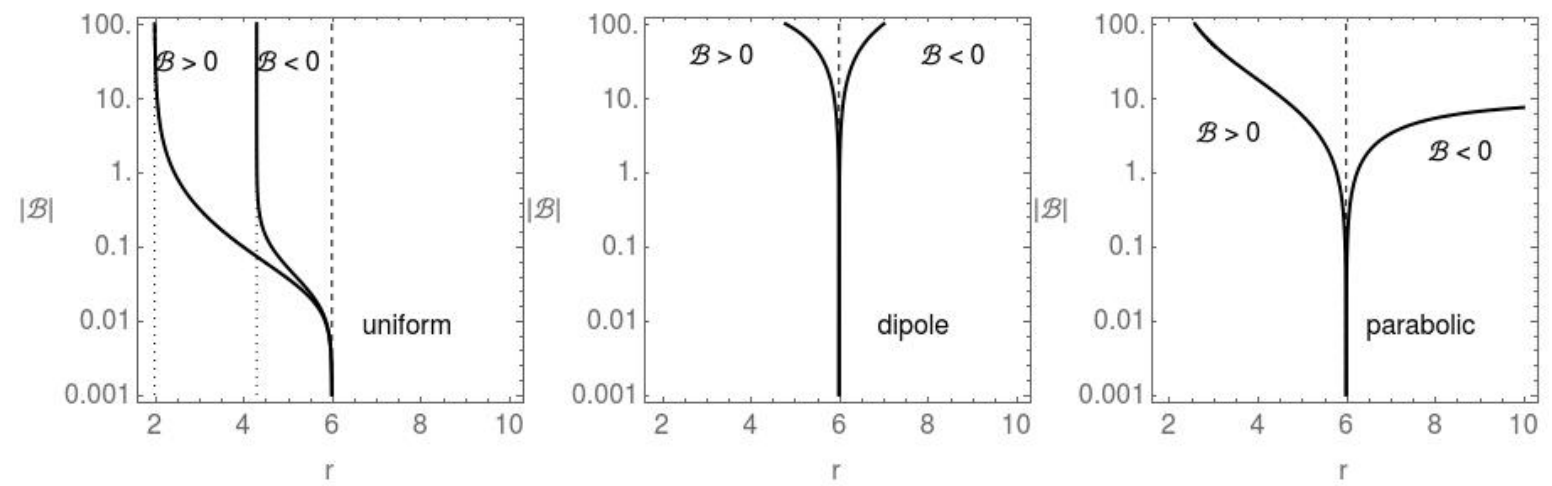

Figure 5. The radii of the charged particle ISCO in dependence on the magnetic field parameters $\mathcal{B}, \mathcal{K}$ are given for the uniform dipole and parabolic magnetic fields. Repulsive Lorentz force $\mathcal{B}>0$ / attractive Lorentz force $\mathcal{B}<0$. Notice the special character of the uniform field case. caused by the fact that the electromagnetic interaction parameter remains independent of the radius, only the velocity of the motion is modified.

\subsection{Fate of Ionized Keplerian Disks Orbiting Schwarzschild Black Holes}

In the first step of our discussion, we consider a Keplerian accretion disk consisting from electrically neutral test particles following circular geodesics and orbiting a Schwarzschild black hole immersed in an asymptotically uniform magnetic field, dipole field or parabolic field, assuming the symmetry plane of the fields almost coincides with the equatorial plane of the Schwarzschild geometry where the location of the Keplerian disk is assumed (Figures 6-8). In the second step of our discussion we consider a Keplerian disk around a Schwarzschild black hole immersed in one of the three considered types of the magnetic field, having an arbitrary inclination angle $\theta$ relative to the magnetic field lines that are perpendicular to the spacetime equatorial plane $\theta=\pi / 2$-see Figure 9 . The case of Keplerian disks inclined to the external uniform magnetic field aligned with the $z$ axis (vertical direction) [5] was considered in detail by [24]. The conditions at the moment of the ionization of the Keplerian disk are thus determined for the case of the arbitrary inclination of the disk in the uniform magnetic field or any other magnetic field, as they can be considered as fully local conditions that can be applied for the ionization event. In the Schwarzschild spacetimes the setup applied in our paper is equivalent to the Keplerian disk located in the spacetime equatorial plane, while the magnetic field lines are inclined to the $z$ axis [25].

To demonstrate the influence of the magnetic field on the ionized Keplerian disk, some realistic ionization scenarios for the originally electrically neutral matter of the disk have to be assumed. As ionization model of the originally neutral matter (particle), we can consider the magnetic Penrose process (MPP) $[9,26]$ where the original 1st neutral particle splits into two oppositely charged particles-2nd and 3rd. We thus assume conservation of the electric charge $0=q_{2}+q_{3}$, and the canonical momentum

$$
\pi_{\alpha(1)}=\pi_{\alpha(2)}+\pi_{\alpha(3)} .
$$

In this case the momentum conservation takes (due to charge conservation) the form

$$
p_{\alpha(1)}=p_{\alpha(2)}+q_{2} A_{\alpha}+p_{\alpha(3)}+q_{3} A_{\alpha}=p_{\alpha(2)}+p_{\alpha(3)} .
$$

In many realistic scenarios, like neutron $\beta$ decay or neutral atom ionization, one of the new created charged particles is much more massive than the other newly created charged particle, $m_{2} / m_{3} \gg 1$, see proton/electron or ion/electron mass ratios. The more massive charged particle (proton or ion) takes almost all the initial momentum of the original neutral particle, and the dynamical influence of the lighter charged particle (electron) can be neglected

$$
p_{\alpha(1)} \approx p_{\alpha(2)} \gg p_{\alpha(3)} \text {. }
$$


In another realistic scenario, we can consider the Keplerian disk created by plasma modelled as a quasi-neutral soup of charged particles: electrons and ions, which are orbiting around the central object on circular orbits. If the disk is dense enough, the main free path of charged particles is much shorter in comparison to the length of the orbit around the central object. This means that the charged particles orbit the black hole together as a collective neutral body along circular geodesics as the influence of the magnetic field is irrelevant for the motion, but at the edges of the disk its density decreases substantially and the charged particle motion starts to be significantly influenced by the magnetic field as the mean free path becomes comparable to the orbit extension.

Both scenarios presented above give the simple ionization model, where the neutral particle will just obtain charge while its mechanical momentum will be conserved. Such an ionization model (19) was already studied in the field of rotating Kerr black hole [4,27], for the special case where the charged particle escape velocities and structure of the escape zones were explored. Here we discuss the fate of the ionized Keplerian disk around magnetized Schwarzschild black holes under general conditions, with special attention devoted to the dependence of the fate on the initial inclination of the disk to the magnetic field lines. Chaoticity of the motion of the ionized matter orbiting Schwarzschild black holes has been recently studied in detail in [24].

Considering the simple ionization model (19), the test particle before (I) and after (II) ionization fulfills the conditions of mass and kinetic momentum conservation

$$
m_{(\mathrm{I})}=m_{(\mathrm{II})}, \quad p_{(\mathrm{I})}^{\mu}=p_{(\mathrm{II})}^{\mu} .
$$

The neutral or charged test particle will be originally located on inclined circular orbit with initial position $x^{\alpha}$ and four-velocity $u_{\alpha}$

$$
\begin{aligned}
& x^{\alpha}=(t, r, \theta, \phi)=\left(0, r_{0}, \theta_{0}, 0\right), \\
& u_{\alpha}=\left(u_{t}, u_{r}, u_{\theta}, u_{\phi}\right)=(\mathcal{E}, 0,0, \mathcal{L}) .
\end{aligned}
$$

Specific axial angular momentum $\mathcal{L}$ and specific energy $\mathcal{E}$ for neutral test particles on the inclined circular orbits from Kepplerian accretion disk are given by [28]

$$
\mathcal{L}_{(\mathrm{I})}=\frac{r_{0} \sin \theta_{0}}{\sqrt{r_{0}-3}}, \quad \mathcal{E}_{(\mathrm{I})}=\frac{r_{0}-2}{\sqrt{r_{0}^{2}-3 r_{0}}} .
$$

Due to the simple ionization condition (20), and using the definitions of the specific energy and specific axial angular momentum (23), we can write for the specific axial angular momentum $\mathcal{L}$ and the specific energy $\mathcal{E}$ of the ionized test particle

$$
\mathcal{L}_{(\mathrm{II})}=\mathcal{L}_{(\mathrm{I})}+\mathcal{B} r_{0}^{2} \sin ^{2} \theta_{0}, \quad \mathcal{E}_{(\mathrm{II})}=\mathcal{E}_{(\mathrm{I})} .
$$

The uniform magnetic field has in the Schwarzschild metric only one non-vanishing component $A_{\phi}(3)$, hence only the specific angular momentum $\mathcal{L}$ is changed during the ionization, while the particle specific energy $\mathcal{E}$ remains constant. The energy of the neutral particles on the circular geodesic orbits, given by (23), is always $\mathcal{E} \leq 1$, but energy $\mathcal{E}>1$ is needed for the charged particle to escape to infinity along the $z$-axis. Therefore, no escape to infinity of neutral matter from Keplerian disks is possible in the Schwarzschild spacetime [4]. We thus have four possibilities of the fate of the ionized Keplerian disk (its inner region near ISCO):

(A) survival in regular epicyclic motion

(B) transformation into chaotic toroidal state

(C) destruction due to fall into $\mathrm{BH}$

(D) destruction due to escape along magnetic field lines 
Since in the Schwarzschild metric the ionized particle following originally a circular geodesic cannot escape to infinity, the escape of ionized particles along the magnetic field lines corresponds to winds rather to jets; the latter case is possible only in the case of the Kerr metric where even very strong acceleration of particles is possible as shown in $[4,14]$. The other possibilities are the same both for the Schwarzschild and Kerr black holes: the capture by the black hole, or bound motion in a regular (epicyclic) regime (in sufficiently weak field), or in fully chaotic regime leading to geometrically thick, toroidal accretion structures (in appropriately strong fields).

We first discuss behavior of the ionized Keplerian disks that are near-perpendicular to the magnetic field lines of the uniform field and in the symmetry plane of the dipole and parabolic magnetic fields, then the inclined disks in the uniform field.

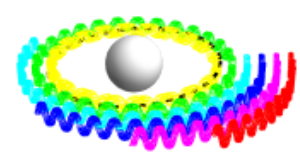

$\mathcal{B}=-1$

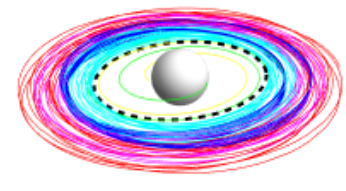

$\mathcal{B}=-0.001$

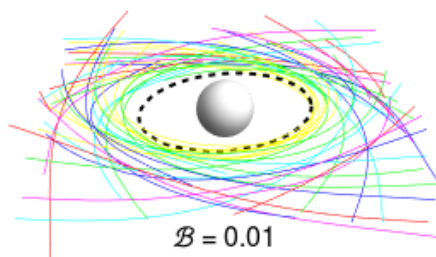

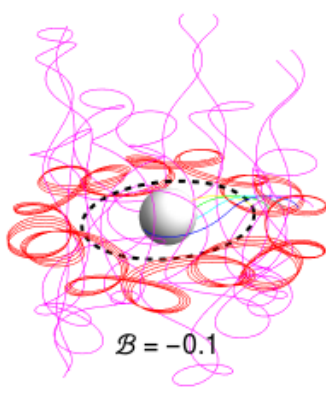

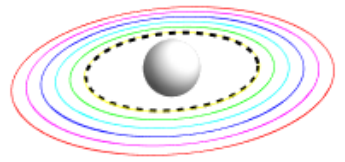

$\mathcal{B}=0$

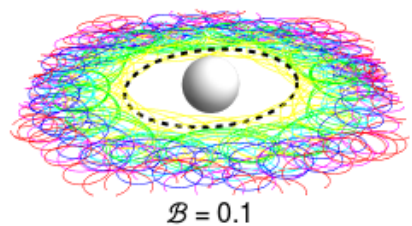

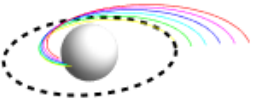

$\mathcal{B}=-0.01$
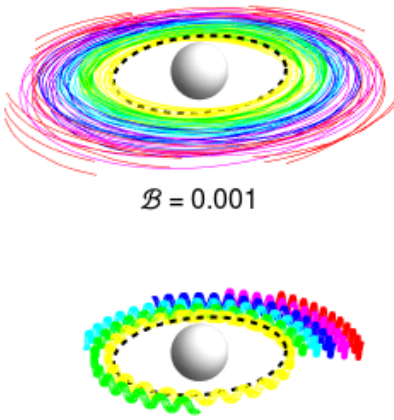

$\mathcal{B}=1$

Figure 6. Thin Keplerian accretion disk around Schwarzschild BH in uniform magnetic field, created by neutral test particles following circular geodesics, and its evolution when the influence of the magnetic field is switched on. The accretion disk particles following initially circular orbits with slight inclination from equatorial plane $\left(\theta_{0}=1.5\right)$, while the magnetic field lines are everywhere aligned with the $z$ axis (vertical direction). The uncharged test particles on innermost stable spherical orbit (depicted by the dashed circle), represent the inner edge of the Keplerian accretion disk. If the disk will remain neutral or the magnetic field is missing ( $\mathcal{B}=0$ case, middle figure) all the orbits will remain in their circular shape and we see just inclined razor thin disk. If a slightly strong electromagnetic interaction is switched-on ( $\mathcal{B}= \pm 0.001$ cases, middle row), the charged particles forming the disk that is originally almost perpendicular to the magnetic field lines start to follow epicyclic oscillations around the circular orbit in both radial and latitudinal directions; the accretion disk becomes to be slightly thick. If larger magnetic field is switched-on ( $\mathcal{B}= \pm 0.01, \pm 0.1$ cases), the charged particle motion becomes quite chaotic and the accretion disk is destroyed or transformed into thick toroidal structure. The complete destruction of the Keplerian disk can be seen in the $\mathcal{B}=-0.01$ case, when all the particles are captured by the black hole. If the magnetic parameter of the field that is switched-on is large $(|\mathcal{B}| \geq 1$ cases), the Lorentz force dominates the particle motion. The charged particles are spiraling up and down along the magnetic field lines, while slowly moving around the black hole in the clockwise $(\mathcal{B}>0)$ or the counter-clockwise $(\mathcal{B}<0)$ direction. The thin Keplerian disk has been destroyed and transformed into some special thick toroidal structure. 


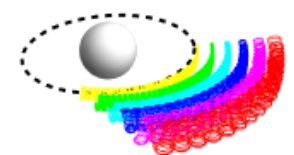

$\mathcal{B}=-1$

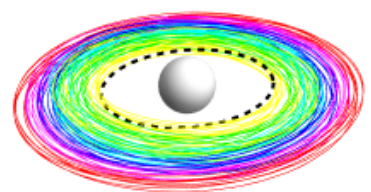

$\mathcal{B}=-0.001$

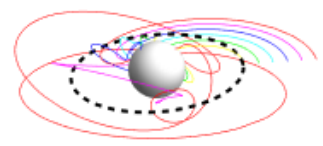

$\mathcal{B}=0.01$
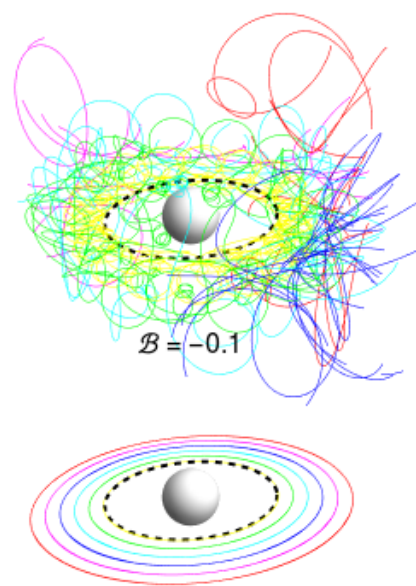

$\mathcal{B}=0$

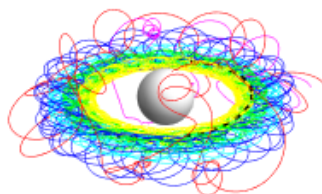

$\mathcal{B}=0.1$
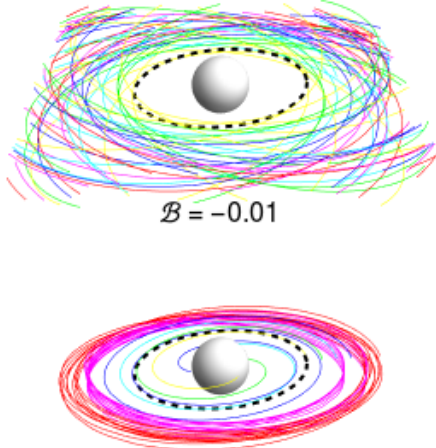

$\mathcal{B}=0.001$

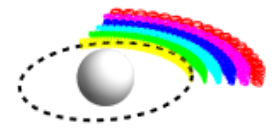

$\mathcal{B}=1$

Figure 7. Thin Keplerian accretion disk around Schwarzschild $\mathrm{BH}$ in dipole magnetic field. and its evolution after ionization. The disk is located closely to the equatorial plane of the background. The dependence of the fate of the ionized disk on the intensity of the electromagnetic interaction (magnetic parameter) is generally in concordance with the case of the uniform magnetic field.

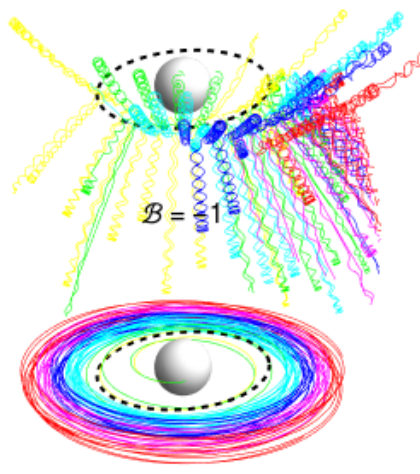

$\mathcal{B}=-0.001$

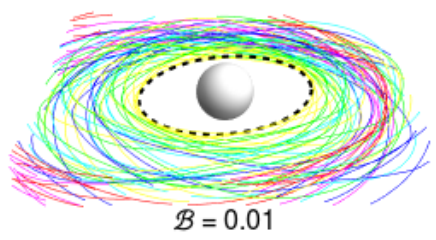

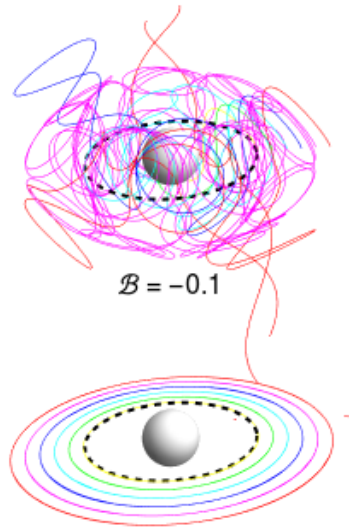

$\mathcal{B}=0$

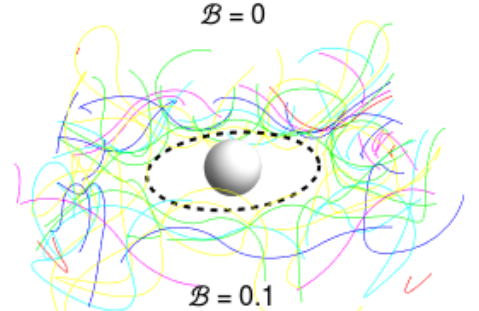

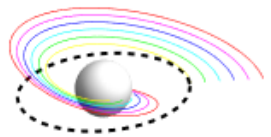

$\mathcal{B}=-0.01$
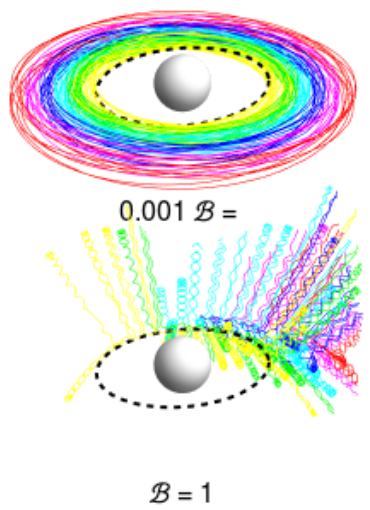

Figure 8. Thin Keplerian accretion disk around Schwarzschild BH in parabolic magnetic field. The disk is located closely to the equatorial plane of the background. The dependence of the fate of the ionized disk on the magnetic parameter is generally in concordance with the case of the uniform and dipole magnetic fields. 


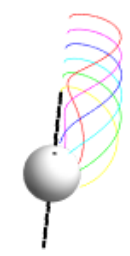

$\theta_{0}=0.1$

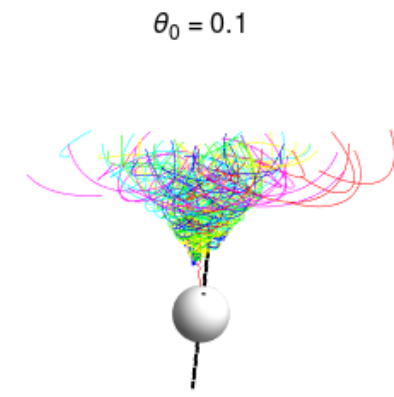

$\theta_{0}=0.1$

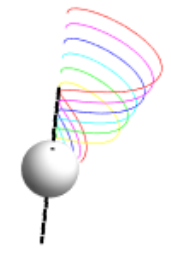

$\theta_{0}=0.1$
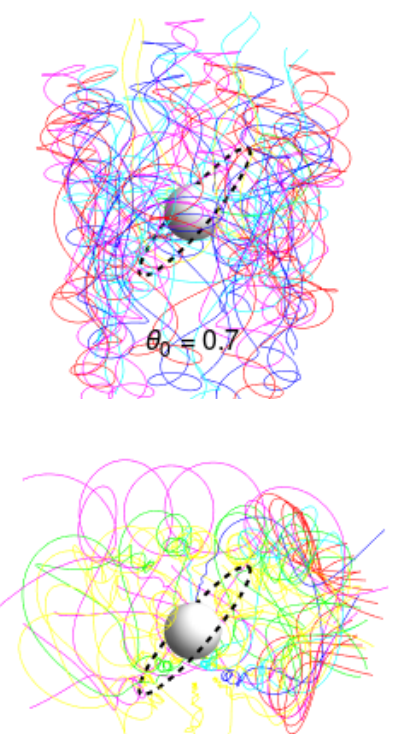

$\theta_{0}=0.7$

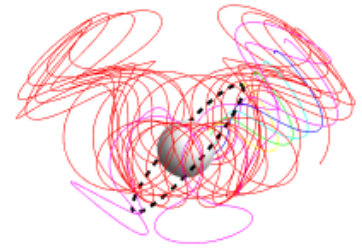

$\theta_{0}=0.7$
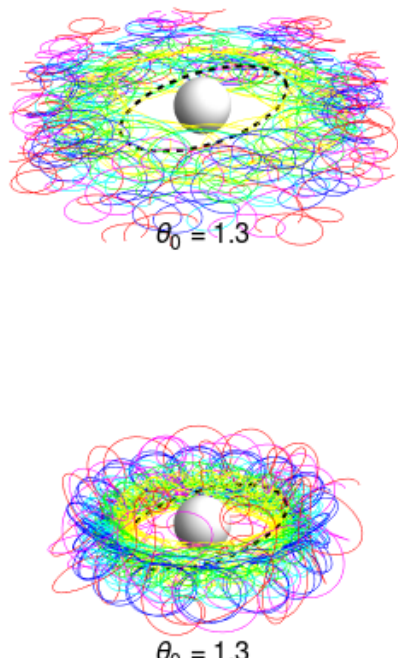

$\theta_{0}=1.3$

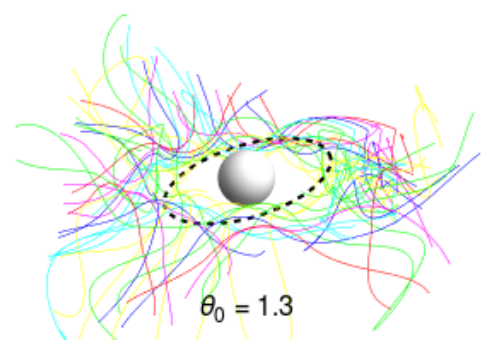

Figure 9. Thin Keplerian accretion disk around Schwarzschild BH in uniform (fist row), dipole (second row) and parabolic (third row) magnetic field. Magnetic field parameter is the same in all cases $\mathcal{B}=0.1$, while the disk inclination $\theta_{0}$ is changing.

\subsubsection{Near-Perpendicular Keplerian Disks in Uniform Magnetic Fields}

The influence of the magnetic field parameter $\mathcal{B}$ on the fate of the Keplerian disks orbiting a Schwarzschild black hole in position that is nearly perpendicular to the magnetic field lines of uniform magnetic field is demonstrated in Figure 6.

When the disk remains neutral or the magnetic field is missing $(\mathcal{B}=0$ case), all the orbits remain circular-original inclined razor thin disk survives. For very small magnetic parameters $(\mathcal{B}= \pm 0.001$ cases), the charged particles forming the disk start to be unsettled and the original circular orbits are perturbed, entering generally epicyclic motion in both radial and vertical directions. Only in the attractive case, the innermost orbits are captured by the black hole. The ionized disk becomes slightly thick due to the epicyclic motion of the charged matter.

When the magnetic field parameter magniture is enlarged, ( $\mathcal{B}= \pm 0.01$ cases), complete destruction of of the inner part of the Keplerian disk occurs in the attractive $\mathcal{B}=-0.01$ case, as all the orbits end at the black hole. In the repulsive $\mathcal{B}=0.01$ case, the regular epicyclic motion has tendency to chaoticity and the ionized disk becomes thick. Further increasing of the magnetic field parameter ( $\mathcal{B}= \pm 0.1$ cases) implies a significant role of the Lorentz force and the charged particle motion enters fully chaotic regime causing a substantial thickening of the disk. Moreoever, especially in the attractive case $\mathcal{B}=-0.1$, we observe trajectories corresponding to winds related to the disk, i.e., trajectories leaving to large distances the structure forming the thick disk - this kind of behaviour is typical for the 
chaotic regime where the energy of the orbital motion can be efficiently transformed to the energy of the vertical motion as demonstrated in [4].

For large values of the magnetic field parameter $(\mathcal{B}= \pm 1$ cases $)$, the Lorentz force becomes the leading force of the particle motion. The charged particles wind up and down along magnetic field lines, while realizing epicyclic motion around black hole in clockwise $(\mathcal{B}>0)$ or counter-clockwise $(\mathcal{B}<0)$ direction. Again, the thin Keplerian disk is transformed into a thick structure around the equatorial plane. For larger values of magnetic field parameter, $|\mathcal{B}|>1$, similar behavior is observed.

\subsubsection{Keplerian Disks in Symmetry Plane of Dipole Magnetic Fields}

The influence of the magnetic field parameter $\mathcal{B}$ on the fate of the Keplerian disks orbiting a Schwarzschild black hole in position corresponding to the symmetry plane of dipole magnetic field is demonstrated in Figure 7. We can see that the dependence on the magnetic parameter $\mathcal{B}$ is of the same character as in the case of the uniform magnetic field, with some special signatures of the dipole field. Generally, the dipole field decreases the signatures of the chaotic character of the motion as demonstrated in the case of $\mathcal{B}= \pm 0.1$ and keeps the special kind of regularity for strong magnetic parameters (cases $\mathcal{B}= \pm 1$ ). For the case of very weak magnetic fields with $\mathcal{B}=-0.001$ we observe increasing tendency to capturing of particles from more distant regions of the disks by the black hole.

\subsubsection{Keplerian Disks in Symmetry Plane of Parabolic Magnetic Fields}

The influence of the magnetic field parameter $\mathcal{B}$ on the fate of the Keplerian disks orbiting a Schwarzschild black hole in position corresponding to the symmetry plane of parabolic magnetic field is demonstrated in Figure 8. The dependence on the magnetic parameter $\mathcal{B}$ is of the same character as in the case of the uniform magnetic field, but it is strongly enlarged in comparison with the bahaviour in the case of the uniform magnetic field. Generally, in the case of very weak and weak magnetic parameters $(\mathcal{B}= \pm 0.001$ and $\mathcal{B}= \pm 0.01)$ the case of the uniform field is very similar. For mediate and strong magnetic field parameters $(\mathcal{B}= \pm 0.1$ and $\mathcal{B}= \pm 1)$ we observe clear signatures of the parabolic character of the magnetic field, even in the case of the chaotic regime.

\subsubsection{Inclined Keplerian Disks in Magnetic Field}

The dependence of the fate of the Keplerian disk on its inclination angle $\theta$ to the magnetic field lines of an uniform magnetic field is studied in detail in [24] — for the mediatelly strong magnetic field parameter $|\mathcal{B}|=0.1$ representing the reachest variety of possible behaviour we can summarize the following conclusions. For small inclination angles $\left(0<\theta<30^{\circ}\right)$ there is a tendency to destroy the full inner region of the disk by its capture by the black hole, for mediate values of the inclination $\left(30^{\circ}<\theta<75^{\circ}\right)$ the chaotic regime fully enters the play creating thick accretion structures, while in the case of large inclination angles corresponding to disks nearly perpendicular to the magnetic field lines $\left(75^{\circ}<\theta<90^{\circ}\right)$, the chaotic regime allows for some exception of regularity corresponding to epicyclic motion. Detailed study of the distribution of the chaoticity and regularity regions in dependence on the parameter $\mathcal{B}$, the position of the original circular geodesic orbit, and its inclination, studied by a variety of non-linear methods of determination of chaotic motion, can be found in [24]. In Figure 9 we add an overview and comparison of the influence of the inclination angle in the case of the uniform, dipole and parabolic magnetic fields.

\section{Ionized Keplerian Disks around Magnetized Rotating Black Holes}

Now we extend our discussion to the fate of ionized Keplerian disks orbiting rotating black holes decribed by the Kerr geometry. In this case, the Keplerian disks have to be located at the equatorial plane of the spacetime geometry or to be only very slightly inclined to this plane (the Bardeen-Petterson effect [13]). For simplicity we assume the magnetic field lines to be orthogonal to the geometry equatorial plane. The black hole spin influences significantly the motion of charged particles in the combined gravomagnetic field. Despite the subtleties of the motion of charged particles around 
magnetized spinning black holes, we are able to show that there remain the four types of the fate of ionized Keplerian disks, established in the case of non-rotating Schwarzschild black holes, with one extension in the case of the disks destroyed due to escape, as in this case the escaping particles could escape to infinity. The reason for this modification is in the presence of the time component of the magnetic field potential causing variation of the particle energy in the MPP [4]. Detailed study of the fate of disks orbiting magnetized Kerr black holes is under preparation and here we report two cases of special astrophysical interest: the regime of the regular epicyclic motion that can be related to the high-frequency quasiperiodic oscillations (HF QPOs), and the regime of efficient acceleration along magnetic filed lines that can be related to creation of ultra-high-energy cosmic rays (UHECR).

\subsection{Magnetized Kerr Black Holes}

The Kerr spacetime is governed by the geometry with line element that in the standard Boyer-Lindquist coordinates takes the form

$$
\mathrm{d} s^{2}=g_{t t} \mathrm{~d} t^{2}+2 g_{t \phi} \mathrm{d} t \mathrm{~d} \phi+g_{\phi \phi} \mathrm{d} \phi^{2}+g_{r r} \mathrm{~d} r^{2}+g_{\theta \theta} \mathrm{d} \theta^{2}
$$

where

$$
\begin{array}{r}
g_{t t}=-\left(1-\frac{2 M r}{\Sigma}\right), \quad g_{t \phi}=-\frac{2 a M r \sin ^{2} \theta}{\Sigma}, \quad g_{\phi \phi}=\left(r^{2}+a^{2}+\frac{2 a^{2} M r}{\Sigma} \sin ^{2} \theta\right) \sin ^{2} \theta \\
g_{r r}=\frac{\Sigma}{\Delta}, \quad g_{\theta \theta}=\Sigma, \quad \Delta=r^{2}-2 M r+a^{2}, \quad \Sigma=r^{2}+a^{2} \cos ^{2} \theta,
\end{array}
$$

$M$ is the gravitational mass of the black hole and $a=J / M$ is its spin; $J$ is its the angular momentum.

The external, asymptotically uniform magnetic field of intensity $B$ with lines oriented along the $z$-axis, i.e., orthogonal to the geometry equatorial plane, is governed by the electromagnetic 4 -vector potential $A_{\alpha}$ with two nonzero components having the form

$$
A_{t}=\frac{B}{2}\left(g_{t \phi}+2 a g_{t t}\right)-\frac{Q}{2} g_{t t}-\frac{Q}{2}, \quad A_{\phi}=\frac{B}{2}\left(g_{\phi \phi}+2 a g_{t \phi}\right)-\frac{Q}{2} g_{t \phi}
$$

where an induced electric charge of the black hole $Q$ is also introduced. For non-charged black holes there is $Q=0$. The maximal induced black hole charge generated by the black hole rotation takes the Wald value of $Q_{W}=2 a B M$ (see [5]). For the black holes having the maximal Wald charge the electromagnetic potential reduces to the form

$$
A_{t}=\frac{B}{2} g_{t \phi}-\frac{Q_{W}}{2}, \quad A_{\phi}=\frac{B}{2} g_{\phi \phi}
$$

It is crucial that even in this case the $A_{t}$ component remains non-zero and can lead to very strong acceleration mechanism for sufficiently massive black holes and strong magnetic fields. In the following we restrict attention to the case with the maximal Wald induced charge $Q_{W}=2 a B M$ as it is astrophysically the most relevant case.

\subsection{Equations of Motion-Hamiltonian Formalism and Effective Potential of the Motion}

Motion of charged test particles, governed by the Lorentz equation, will be treated in the Hamiltonian formalism, as in the case of the Schwarzschild black holes. The basic equations are presented in previous section.

The stationarity and axial symmetries of the background, corresponding to the magnetized Kerr black holes with the magnetic field lines orthogonal to the equatorial plane of the spacetime geometry, imply that the charged test particle motion can be again reduced to the two-dimensional dynamics, as we can introduce two constants of the motion: energy $E$ and angular momentum $L$, given by the conserved componets of the canonical momentum 


$$
-E=\pi_{t}=g_{t t} p^{t}+g_{t \phi} p^{\phi}+q A_{t}, \quad L=\pi_{\phi}=g_{\phi \phi} p^{\phi}+g_{\phi t} p^{t}+q A_{\phi} .
$$

For further discussion, it is convenient to introduce the specific energy $\mathcal{E}=E / m$, the specific axial angular momentum $\mathcal{L}=L / m$, and the magnetic interaction parameter $\mathcal{B}=q B / 2 m$. Then we obtain, in close analogy with the case of the motion around magnetized Schwarzschild black holes, Hamiltonian with two degrees of freedom, and $4 \mathrm{D}$ phase space $\left(r, \theta ; p_{r}, p_{\theta}\right)$ of the motion of charged particles; the Hamiltonian can be again expressed in the form

$$
H=\frac{1}{2} g^{r r} p_{r}^{2}+\frac{1}{2} g^{\theta \theta} p_{\theta}^{2}+\widetilde{H_{\mathrm{P}}}(r, \theta)
$$

that enables introduction of the effective potential of the radial and latitudinal motion giving the energetic boundary for the particle motion due to the condition $\widetilde{H_{\mathrm{P}}}=0$. The energy condition relates the specific energy to the effective potential $\mathcal{E}=V_{\text {eff }}(r, \theta)$ defined in the case of the Kerr black holes by

$$
V_{\mathrm{eff}}(r, \theta)=\frac{-\beta+\sqrt{\beta^{2}-4 \alpha \gamma}}{2 \alpha}
$$

with

$$
\alpha=-g^{t t}, \quad \gamma=-g^{\phi \phi}\left(\mathcal{L}-\tilde{q} A_{\phi}\right)^{2}-g^{t t} \tilde{q}^{2} A_{t}^{2}+2 g^{t \phi} \tilde{q} A_{t}\left(\mathcal{L}-\tilde{q} A_{\phi}\right)-1
$$

\subsection{Circular Orbits and Related Epicyclic Motion}

The motion of charged particles in the field of magnetized black holes has generally chaotic character. However, there exists an important exception, namely the circular motion in the equatorial plane, and the associated epicyclic motion in the equatorial plane and its vicinity [6,7]. The circular orbits of charged test particles exist in the equatorial plane, being determined by the local extrema of the effective potential governed by the relations $\partial_{r} V_{\text {eff }}=0$ and $\partial_{\theta} V_{\text {eff }}=0$. It has been shown that the circular orbits can be separated into four classes: Prograde anti-Larmor orbits (PALO) where the particle is corotating $(\mathcal{L}>0)$, magnetic lines are co-oriented with the black hole rotation axis $\mathcal{B}>0$, Lorentz force is repulsive; Retrograde Larmor orbits (RLO) with the particle counter-rotating $(\mathcal{L}<0)$, magnetic lines co-oriented with the black hole rotation axis $(\mathcal{B}>0)$, Lorentz force attractive; Prograde Larmor orbits (PLO) with $\mathcal{L}>0, \mathcal{B}<0$, Lorentz force attractive; Retrograde anti-Larmor orbits with $\mathcal{L}<0$, $\mathcal{B}<0$, Lorentz force repulsive. Notice that for the Schwarzschild black holes, classes PALO/RALO, and RLO/PLO coincide. The circular motion is stable against radial (latitudinal, i.e., vertical) perturbations if $\partial_{r}^{2} V_{\text {eff }}<0\left(\partial_{\theta}^{2} V_{\text {eff }}<0\right)$, otherwise the circular motion is unstable. The vanishing of the second derivative of the effective potential corresponds to the innermost (marginally) stable circular orbit. Detailed analysis of the circular orbits in the background of magnetized Kerr black holes can be found in [7].

The stable circular orbits correspond to the minima of the effective potential, i.e., for given specific angular momentum $\mathcal{L}$ the circular orbits has minimal specific energy $\mathcal{E}$. If the particle specific energy slightly exceeds, for a given $\mathcal{L}$, the minimum corresponding to the stable circular orbit, the particle enters regime of regular epicyclic motion. The epicyclic motion has in the basic approximation character of the linear harmonic oscillations, as implied by the following simple demonstration. Assuming motion in the $x$-direction, the general equation of the motion takes the form $\ddot{x}=F / m=-V_{x}^{\prime}$. In order to describe variations of the position $\delta x=x-x_{0}$ around a stationary stable point $x_{0}$, where $V_{x}^{\prime}\left(x_{0}\right)=$ 0 , we use the Taylor expansion of the potential $V(x)=V\left(x_{0}\right)+V_{x}^{\prime}\left(x_{0}\right) \delta x+1 / 2 V_{x}^{\prime \prime}\left(x_{0}\right) \delta x^{2}+\ldots$ that implies for the perturbation of the stationary stable position the linear oscillation equation $\ddot{\delta} x+V_{x}^{\prime \prime}\left(x_{0}\right) \delta x=0$, or $\ddot{\delta x}+\omega_{x}^{2} \delta x=0$ where $\omega$ denotes angular frequency of the harmonic oscillations. The frequencies of the charged particle epicyclic radial and latitudinal motion in vicinity of the circular orbit located at a minimum of the effective potential $V_{\text {eff }}(r, \theta)$ of the magnetized Kerr black hole can be thus given by the relations [7] 


$$
\omega_{r}^{2} \sim \frac{\partial^{2} V_{\text {eff }}}{\partial r^{2}}, \quad \omega_{\theta}^{2} \sim \frac{\partial^{2} V_{\text {eff }}}{\partial \theta^{2}} .
$$

Here we demonstrate a straightforward way of giving the frequency of the epicyclic motion around magnetized Kerr black holes using directly perturbations of the Lorentz equations of motion [8]. The four-velocity of the circular orbits has two non-zero components, $u^{\mu}=\left(u^{t}, 0,0, u^{\phi}\right)$. For the maximally charged Kerr black holes (with the Wald charge $Q_{W}$ ), the Lorentz equation implies for the radial component of the equatorial motion

$$
\left(a^{2}-r^{3}\right)\left(u^{\phi}\right)^{2}-2 \mathcal{B}\left(r^{3}-a^{2}\right) u^{\phi}-2 a\left(u^{\phi}+\mathcal{B}\right) u^{t}+\left(u^{t}\right)^{2}=0
$$

and the normalization condition for the four-velocity implies

$$
\left(a^{2}(2+r)+r^{3}\right)\left(u^{\phi}\right)^{2}-4 a u^{\phi} u^{t}-(r-2)\left(u^{t}\right)^{2}+r=0 .
$$

These are two simple quadratic equations for two components of the four-velocity. Determination of a circular orbit at a given radius $r$ directly by the four-velocity components $u^{t}$ and $u^{\phi}$ is an alternative to determination by the motion constants $\mathcal{L}$ and $\mathcal{E}$. Then we can directly determine the orbital frequency of the particle relative to distant observers $\Omega_{\phi}$, called also Keplerian fequency $\left(\Omega_{K}\right)$ (or more precisely magnetically modified Keplerian frequency), and compare it to the Larmor frequency $\left(\Omega_{L}\right)$ related purely to the external magnetic field:

$$
\Omega_{\phi} \equiv \Omega_{K}=\frac{d \phi}{d t}=\frac{u^{\phi}}{u^{t}}, \quad \Omega_{L}=\frac{q B}{m u^{t}}=\frac{2 \mathcal{B}}{u^{t}} .
$$

The particle epicyclic motion given by $x^{\mu}(\tau)$, about the "equilibrium" stable circular orbits with $x_{0}^{\mu}(\tau)$, is characterized by deviation vector $\xi^{\mu}(\tau)=x^{\mu}(\tau)-x_{0}^{\mu}(\tau)$. Using the deviation vector in the Lorents equation, and restricting to the first order expansion at small deviations, we arrive to the equations of linear harmonic oscillations in the radial and vertical directions

$$
\frac{d^{2} \xi^{s}}{d t^{2}}+\Omega_{s}^{2} \xi^{s}=0, \quad s \in\{r, \theta\},
$$

where $\Omega_{r}\left(\Omega_{\theta}\right)$ is the angular frequency of the particle radial (latitudinal, i.e., vertical) epicyclic motion measured by distant static observers. For the maximally charged magnetized Kerr black holes we arrive to the relations [8]

$$
\begin{aligned}
& \Omega_{r}^{2}=\frac{\gamma u^{\phi}\left(u^{\phi}+2 \mathcal{B}\right)+v u^{t}\left(u^{\phi}+\mathcal{B}\right)+\rho\left(u^{t}\right)^{2}+\sigma}{r^{5}\left(u^{t}\right)^{2}}, \\
& \Omega_{\theta}^{2}=\frac{\alpha u^{\phi}\left(u^{\phi}+2 \mathcal{B}\right)+\beta u^{t}\left(u^{\phi}+\mathcal{B}\right)+2 a^{2}\left(u^{t}\right)^{2}}{r^{5}\left(u^{t}\right)^{2}},
\end{aligned}
$$

where

$$
\begin{array}{r}
\alpha=r^{5}+a^{2} r^{2}(4+r)+2 a^{4}, \beta=-4 a\left(r^{2}+a^{2}\right), \gamma=r^{4}(-8+3 r)+a^{2} r\left(2-10 r+r^{2}\right)-4 a^{4}, \\
v=4 a\left(2 a^{2}-r+3 r^{2}\right), \rho=2\left(-2 a^{2}+r-r^{2}\right), \sigma=4 \mathcal{B}^{2} r\left((-2+r) r^{3}-a^{2}(1+2 r)\right) .
\end{array}
$$

The fundamental angular frequencies characterizing the orbital and related epicyclic motion of charged particles are direct generalization of their analogues obtained for the pure geodesic motion. They are presented in the dimensionless form. If we want to have directly observable frequencies of the motion given in physical units, we have to use the formula

$$
v_{i}=\frac{c^{3}}{2 \pi G M} \Omega_{i}[\mathrm{~Hz}],
$$

where $i=(\phi, r, \theta)$. 
The radial profiles of the orbital and epicyclic frequencies of the magnetized circular and related epicyclic motion of charged particles demonstrates a variety of fundamental differences in comparison to the frequencies corresponding to the purely geodesic motion of neutral particles, but the crucial effect of vanishing of the radial epicyclic frequency $v_{r}$ at the ISCO holds naturally in both cases [8].

The largest differences are related to the behavior of the frequencies $v_{r}$ and $v_{\phi}=v_{K}$ due to strong effect of the Lorentz force in the radial direction. The magnetic interaction increases the value of the radial epicyclic frequency $v_{r}$, especially for the PALO and RALO orbits, while the axial (Keplerian) frequency $v_{K}$ is strongly decreased for such orbits. On the other hand, the radial profile of the latitudinal (vertical) epicyclic frequency $v_{\theta}$ is not influenced significantly by the magnetic field due to the direction of the Lorentz force.

While for the geodesic motion the relation $v_{r}<v_{\theta}<v_{\phi}$ holds at all the radii of the radial profiles, if we assume non-extreme black holes, in the case of magnetized orbits, the interrelations between the three frequency radial profiles are much more complex, being strongly dependent on the type of the orbit. Nevertheless, the crucial difference is characteristic for all the types of the orbits, namely the crossing of the $v_{r}(r)$ and the $v_{\theta}(r)$ profiles slightly above the ISCO radius. Moreover, in the case of the PALO and RALO orbits, there is also crossing of the $v_{r}$ and the $v_{\phi}$ radial profiles, and in the case of the PALO orbits even the crossing of the $v_{\phi}$ and the $v_{\theta}$ radial profiles is possible.

Effects related to the crossings of the radial profiles of te frequencies $v_{r}(r), v_{\theta}(r)$, and $v_{\phi}(r)$ could have important astrophysical consequences with strong observable signatures, e.g., vanishing of the nodal shift of orbiting matter, etc. Another important effect is related to the RALO orbits where we can observe situations with orbital frequency much smaller than both the epicyclic frequencies, $v_{\phi}<<v_{r} \sim v_{\theta}$. In Figure 10. we demonstrate behaviour of some typical radial frequency profiles. Detailed discussion of the orbital and epicyclic frequencies of the magnetized orbits can be found in $[7,8]$. It is also worth to mention that the frequnecy profiles of the magnetized circular orbits are similar to frequencies of the oscillations of string loop discussed in $[29,30]$.
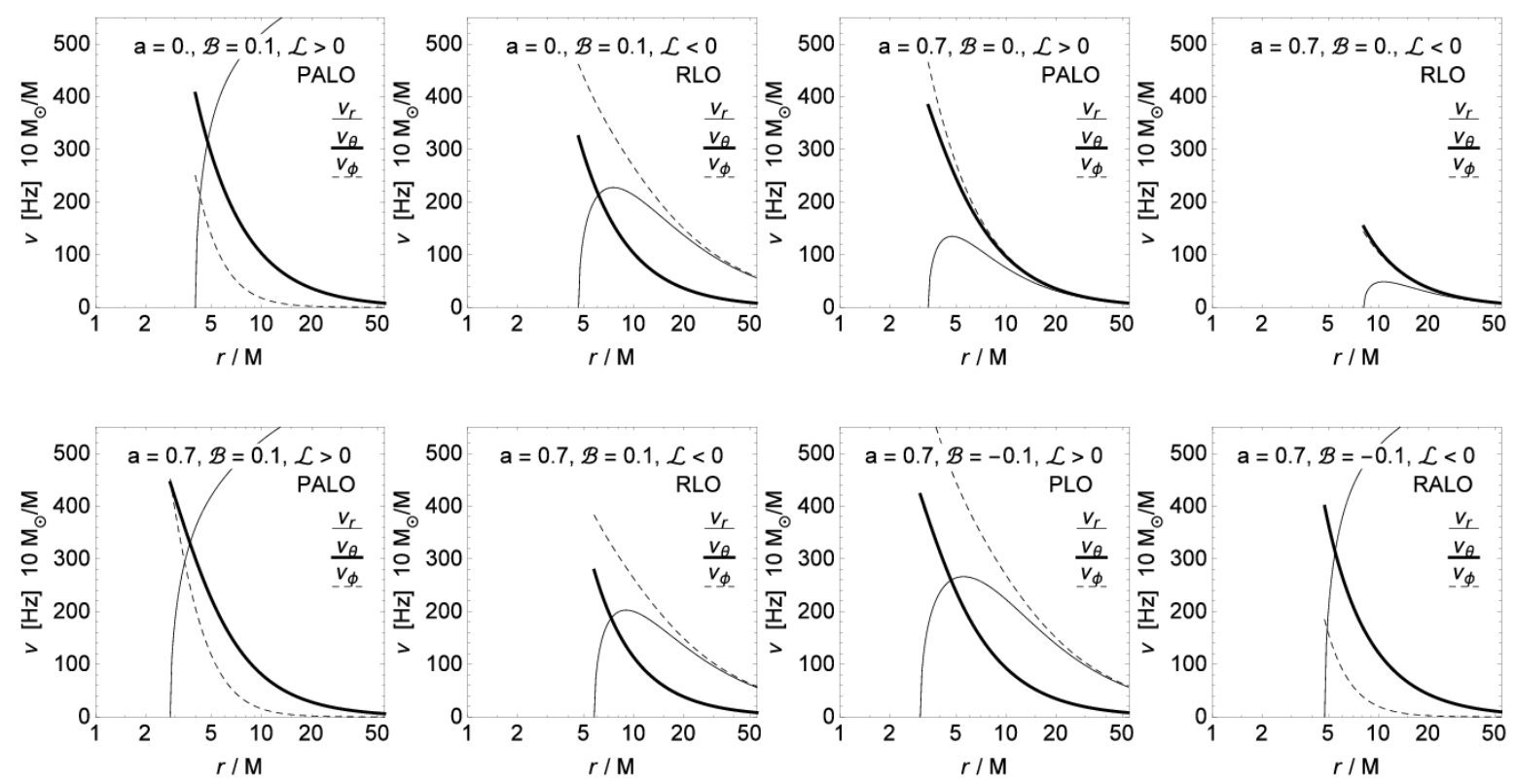

Figure 10. Radial profiles of the frequencies of small harmonic oscillations $v_{\theta}, v_{r}$ and $v_{\phi}$ of charged particle around Kerr black hole with mass $M=10 \mathrm{M}_{\odot}$ and spin $a$ in external magnetic field. The first row represents the influence of magnetic parameter $\mathcal{B}$ in the absence of rotation (first two figures) and rotation in the nonmagnetic case (last two figures). Second row represents the Kerr black hole with $a=0.7$ with magnetic parameter $\mathcal{B}= \pm 0.1$ and Wald charge $Q=Q_{\mathrm{W}}$. 


\subsection{Twin HF QPOs Observed in Microquasars and Their Explanation by Magnetically Modified Geodesic Models}

The frequencies of the orbital and epicyclic geodesic motion play a crucial role in explaining of the twin HF QPOs observed in microquasars, i.e., binary systems composed of a black hole and a companion star. The HF QPOs are observed as peaks of the X-ray power density and are assumed to be created in the innermost parts of the Keplerian accretion disks as the observed frequencies are comparable to the frequency $(v \sim 100-400 \mathrm{~Hz})$ of the orbital Keplerian motion near the ISCO of the stellar mass black holes $\left(M \sim 5-15 \mathrm{M}_{\odot}\right)$ [31]. The HF QPOs in the microquasars are usually detected with the twin peaks which have frequency ratio close to 3:2, indicating relevance of the resonance phenomena [32,33]. Twin HF QPOs of these properties are extensively studied in the case of three microquasars related to the sources GRS 1915+105, XTE 1550-564 and GRO 1655-40. The mass (estimated by optical measurements) and spin (estimated by spectral continuum fitting method measurements [34]) of the black holes related to these microquasars are presented in Table 2. Both these methods are independent of the HF QPO measurements.

Table 2. Observed QPOs data for three microquasars and the restrictions on mass $M$ and spin $a$ of the black holes located in them, based on measurements independent of the QPOs.

\begin{tabular}{lccccc}
\hline Source & $f_{\text {low }}[\mathrm{Hz}]$ & $f_{\mathrm{L}}[\mathrm{Hz}]$ & $f_{\mathrm{U}}[\mathrm{Hz}]$ & $\boldsymbol{M}\left[\mathbf{M}_{\odot}\right]$ & $\boldsymbol{a}$ \\
\hline GRO 1655-40 & 18 & 300 & 450 & $6.03-6.57$ & $0.65-0.75$ \\
XTE 1550-564 & 13 & 184 & 276 & $8.5-9.7$ & $0.29-0.52$ \\
GRS 1915+105 & 10 & 113 & 168 & $10.6-14.4$ & $0.95-1.00$ \\
\hline
\end{tabular}

The observed twin HF QPOs are modelled by a variety of "geodesic" models that assume significant role of the orbital and epicyclic frequencies of the circular geodesic motion and various combinations of these frequencies [35-37]. Due to the frequency ratio 3:2 of the observed frequencies, resonance effects are asumed [32]. The most frequently discussed are the epicyclic resonance [33] and the hot-spot relativistic precession [38] models, having simple identification of the twin observed frequencies (lower $f_{1}$, and upper $f_{\mathrm{u}}$ ) with the frequencies of the geodesic QPO models. In the case of the epicyclic resonance model there is

$$
f_{1}=v_{r}, \quad f_{\mathrm{u}}=v_{\theta},
$$

while in the relativistic precession model there is

$$
f_{1}=v_{\phi}-v_{r}, \quad f_{\mathrm{u}}=v_{\phi} .
$$

For wide variety of modifications of the simple epicyclic and relativistic precession models see $[35,37]$.

It is shown that the geodesic QPO models can explain the twin HF QPOs in all the three considered microquasars, but not by a single version [39]. The magnetic modification of the geodesic QPO models enables improvement of this discrepancy due to the more complex behaviour of the radial profiles of the orbital and epicyclic frequencies. In the procedure of fitting the observational data to the QPO models, we have to compare the calculated frequencies $v_{r}(r, M, a, \mathcal{B}), v_{\theta}(r, M, a, \mathcal{B}), v_{\phi}(r, M, a, \mathcal{B})$, and their combinations corresponding to a specific variant of the QPO model, to the observed QPO frequencies $v_{u}=f_{u}$, and $v_{l}=f_{l}$, obtaining thus restrictions on the parameters $a, M, r, \mathcal{B}$. One of the most important ingredients of the magnetic modification of the geodesic model is presence of the crossing point of the epicyclic radial and latitudinal (or orbital) frequencies. Then we can determine the "resonance" radius $r_{3: 2}\left(r_{2: 3}\right)$ due to resonance conditions

$$
\nu_{\mathbf{u}}\left(r_{3: 2}\right): v_{1}\left(r_{3: 2}\right)=3: 2, \quad v_{\mathbf{u}}\left(r_{2: 3}\right): v_{1}\left(r_{2: 3}\right)=2: 3 .
$$


As the resonance conditions are independent of the black hole mass $M$, the solution of the resonant condition has no dependence on mass and techniques developed in [35] for the geodesic QPO models can be equally applied for the magnetic modifications. Introducing the resonance radius $r_{3: 2}\left(r_{2: 3}\right)$ to the equations for the frequencies $v_{u}$ or $v_{l}$, we express them in terms of the black hole mass, spin, and the magnetic parameter that give so called fitting lines. This enables comparison with limits on the black hole mass and spin obtained by other methods. We have demonstrated that the fitting could be done well both for the epicyclic and the relativistic resonance models. Some results are presented in Figure 11 and Table 3. Details can be found in [8].

Table 3. Magnitude of magnetic filed for three microquasars, obtained by the fitting of the HF QPO frequencies with the frequencies of the oscillations of an electron. We use combined values for the ER model related to the 3:2 and 2:3 resonances (Table 2); similarly for the RP model. All numbers in the table are in units of $10^{-5}$ Gauss for electrons (around $2 \times 10^{-2}$ Gauss for protons).

\begin{tabular}{lccc}
\hline model & GRO 1655-40 & XTE 1550-564 & GRS 1915+105 \\
\hline ER & & & \\
PALO & $11.5-23.0$ & $8.2-16.3$ & $0-14.5$ \\
RLO & $\nexists$ & $21.8-\infty$ & $9.6-\infty$ \\
PLO & $0-61.4$ & $10.9-43.6$ & $0-28.9$ \\
RALO & $23.1-26.9$ & $13.6-16.3$ & $7.2-19.3$ \\
\hline RP & & & \\
RLO & $8.4-13.4$ & $10.6-13.9$ & $3.1-15.7$ \\
\hline
\end{tabular}
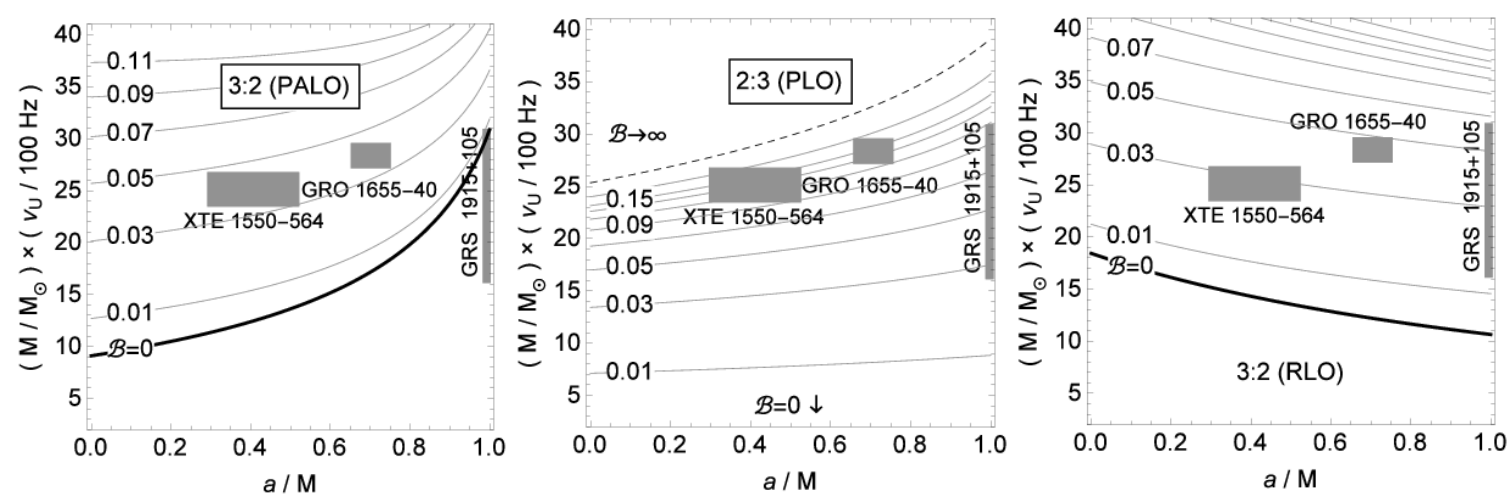

Figure 11. Fitting of three microquasar sources with HF QPOs model of charged particle oscillations with the effect of magnetic field. On the horizontal axis the black hole spin $a$ is given, while on the vertical axis we give black hole mass $M$ divided by observed upper HF QPOs frequency $v_{\mathrm{u}}$. Gray boxes represent the values of black hole mass $M$ and spin $a$ estimated from the observations, see Table 2 . Thin lines are the fitting lines given for various values of the magnetic parameter $\mathcal{B}$. The non-magnetic case $\mathcal{B}=0$ is represented by thick lines. There could exist both 3:2 and 2:3 resonances and four different combinations of the magnetic field/black hole spin configurations, namely PALO, RLO, PLO and RALO, giving thus eight different cases in total, see $[7,8]$.

\subsection{Acceleration of Charged Particles Due to Magnetic Penrose Process}

In vicinity of a magnetized Kerr black hole, the time component of the electromagnetic potential is non-zero enabling acceleration of charged particles-the effective potential of the motion of a charged particle with angular momentum $L$ can be expressed in the form

$$
V_{\mathrm{eff}}=-q A_{t}-\frac{g_{t \phi}}{g_{\phi \phi}} L+\left[\left(-g_{t t}+\frac{g_{t \phi}^{2}}{g_{\phi \phi}}\right)\left(\frac{L^{2}}{g_{\phi \phi}}+1\right)\right] .
$$


The presence of the first term guarantees extension of possible negative energy states accross the ergosphere.

We consider now the same mechanism of the ionization of neutral matter constituting a Keplerian accretion disk as in the case of the Schwarzschild black hole. Thus a neutral 1st particle of mass $m_{1}$ decays into two charged particles (proton and electron)—-their masses satisfy the relation $m_{(1)} \sim$ $m_{(2)} \gg m_{(3)}$ and the kinetic momenta satisfy with high precision the relation $p_{\alpha(1)}=p_{\alpha(2)}$.

The energy of the second particle (e.g., proton) $E_{2}=p_{t 2}+q A_{t}$ can be very large, while energy of the third particle (electron) $E_{3}=p_{t 3}-q A_{t}$ can be negative with very large magnitude (or vice versa, in dependence on the orientation of the magnetic field lines). The process of the chaotic scattering, discussed in [4], guarantees that the highly energetic 2nd particle escapes to infinity along the magnetic field lines; the 3rd particle with large negative energy is immediately captured by the black hole, as demonstrated in Figure 12.

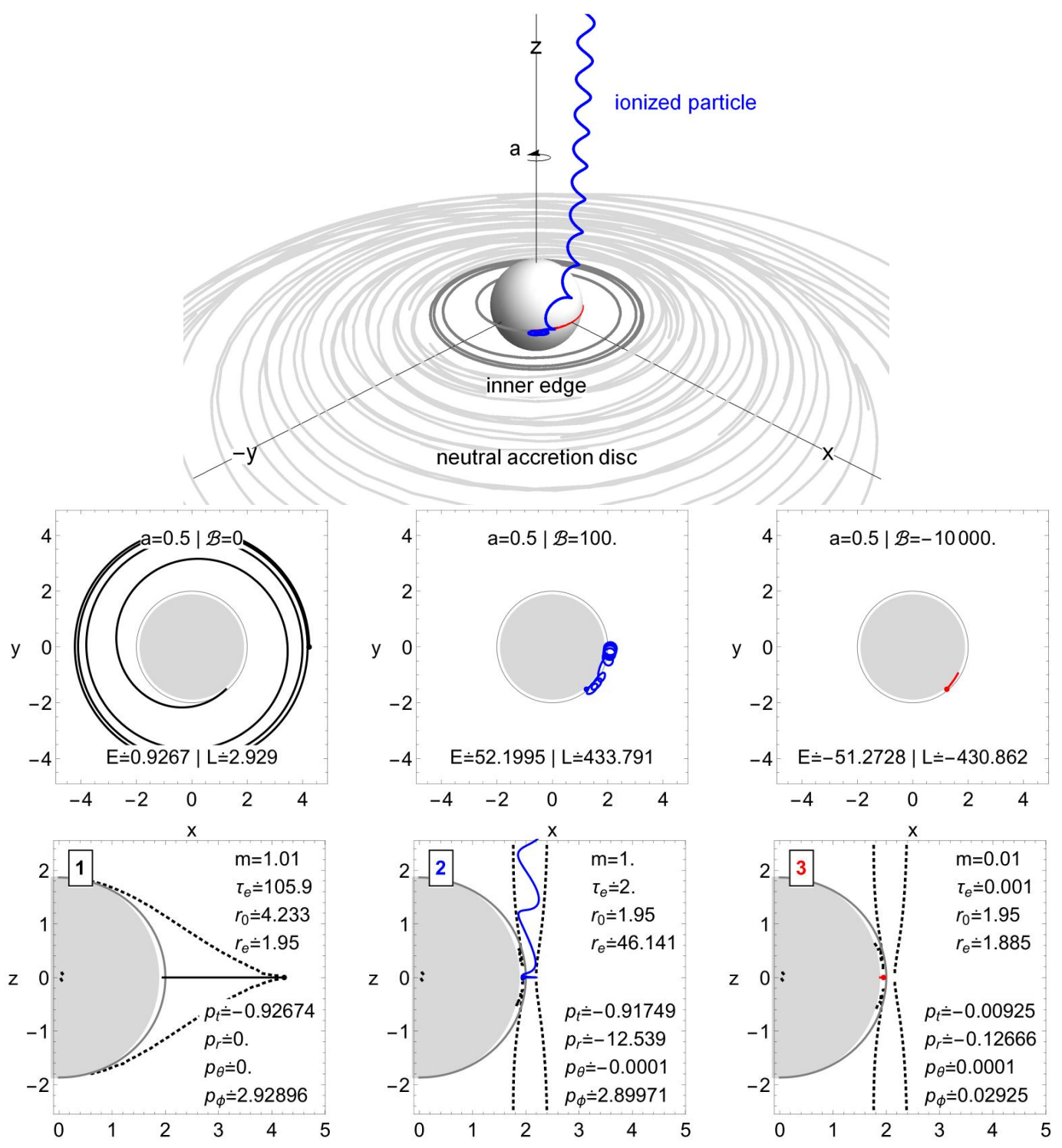

Figure 12. Particle acceleration in vicinity of a magnetized rotating black hole -1 st neutral particle (black, neutron) has been located on accretion disk inner edge, and starts to fall into the black hole, decaying into two charged particles, 2nd (blue, proton), and 3rd (red, electron). While the 3rd particle (red) falls into the black hole with large negative energy, the 2nd (blue) gets large energy and due to the so called chaotic scattering escapes along the magnetic field line. 


\subsection{Efficiency of the Magnetic Penrose Process}

Energy of the second particle can be given by the expression

$$
E_{2}=\chi\left(E_{1}+q_{1} A_{t}\right)-q_{2} A_{t}
$$

with

$$
\chi=\frac{\Omega_{1}-\Omega_{3}}{\Omega_{2}-\Omega_{3}} \frac{X_{2}}{X_{1}}, \quad X_{i}=g_{t t}+\Omega_{i} g_{t \phi}
$$

where $\Omega_{i}=d \phi / d t$ is the angular velocity of the $\mathrm{i}$-th particle. The magnetic Penrose process needs $-q_{2} A_{t}<0$ playing dominant role. For $q_{2}>0$ and $B>0, a>0$ this condition is satisfied.

Using the standard definition of efficiency

$$
\eta=\frac{E_{2}-E_{1}}{E_{1}}=\frac{-E_{3}}{E_{1}}
$$

we arrive at the relation of the efficiency of the magnetic Penrose process in the form

$$
\eta_{\mathrm{MPP}}=\chi-1+\frac{\chi q_{1} A_{t}-q_{2} A_{t}}{E_{1}}
$$

The magnetic Penrose process demonstrates three regimes of efficiency. Those related to the original Penrose process [40] represents the low efficiency regime, when maximal $\eta_{\mathrm{PP}}=\frac{\sqrt{2}-1}{2} \sim 0.207$. When the electromagnetic forces enter the play in dominant way, i.e., $\left|\frac{q}{m} A_{t}\right|>>\left|u_{t}\right|$, we enter the moderate regime with $\eta_{\mathrm{MPP}} \sim \frac{q_{2}}{q 1}-1$ operating while $q_{2}>q_{1}$, neutralizing gravitationally the black hole induced electric field. The third regime, corresponding to ionization of neutral matter, has $\eta_{\text {MPP }} \sim \frac{q_{2}}{m_{1}} A_{t}$, enabling an enormous increase of the efficiency and giving enormous energy to escaping particles. In such a case the efficiency can be as large as $\eta_{\text {MPP }} \sim 10^{9}$. Moreover, for large efficiency of the magnetic Penrose process black hole rotation is necessary, but near-extreme black holes are not necessary, in contradiction to the Blandford-Znajek process.

\subsection{Ultra-High-Energy Cosmic Rays Created by Magnetic Penrose Process}

Cosmic rays are detected with extremely large extend of the energy of particles that are usually high-energy protons or ions; extra-Galactic origin is assumed [41,42]. Observation of the Ultra-High-Energy Cosmic Rays (UHECRs) corresponds to particles having the energy $E>10^{18} \mathrm{eV}$-occassionally, particles with energy $E>10^{21} \mathrm{eV}$ are observed, overcoming the so called GZK limit of $10^{19} \mathrm{eV}$ caused due to interaction with the cosmic microwave background [43]. Observations of particles with energy higher than the GZK limit implies strong restrictions on the distance of the source of such energetic particles.

It is difficult to explain extreme acceleration of particles with energy $E>10^{21} \mathrm{eV}$, but we are able to propose a simple mechanism based on the ultra-efficient regime of the magnetic Penrose process, if it is acting in close vicinity of a supermassive black hole surrounded by sufficiently strong magnetic field.The energy of a charged particle created in the magnetic Penrose process in the extremely efficiency regime can be expressed as

$$
E_{\mathrm{MPP}}=1.3 \times 10^{20} \mathrm{eV} \frac{q}{e} \frac{m_{p}}{m} \frac{a B}{10^{4} \mathrm{G}} \frac{M}{10^{9} \mathrm{M}_{\odot}},
$$

where $q, m$ is test particle charge and mass, while $e, m_{p}$ is proton charge and mass. Clearly, the protons with energy $E>10^{21} \mathrm{eV}$ can be created even by mildly spinning, $a \sim 0.8$, supermassive black holes with mass $M=10^{10} \mathrm{M}_{\odot}$ immersed in magnetic field of intensity $B=10^{4} \mathrm{G}$.

It is also quite interesting that in relation to the supermassive black hole SgrA* and related magnetic field observed in the Galaxy centre, we obtain the proton energy 


$$
E_{\mathrm{p}-\mathrm{SgrA} *}=10^{15.6} \mathrm{eV} \frac{q}{e} \frac{m_{p}}{m} \frac{B}{100 \mathrm{G}} \frac{a}{0.5} \frac{M}{4.14 \times 10^{6} \mathrm{M}_{\odot}}
$$

that coincides with the knee in the observed data corresponding to the significant lowering of number of detected high-energy particles.

\section{Conclusions}

Magnetic field strongly affect astrophysical processes around black holes and other compact objects. If the specific particle charge $q / m$ is large enough, even weak magnetic field can significantly influence position of the inner edge of an ionized Keplerian accretion disc, the charged particle trajectory and charge particle oscillatory frequencies.

We tested charged particle motion in three magnetic filed configurations: uniform, dipole and parabolic. The charged particle ISCO behave differently for dipole/parabolic or uniform field: for dipole and parabolic fields the ISCO is increasing or decreasing with the field strength, depending on direction of Lorentz force; for uniform filed is the ISCO always decreasing. Real magnetic field around black holes and other compact object can significantly differ from completely regular and smooth uniform magnetic field configuration, but any magnetic field can be approximated by uniform at least at small scales. Such magnetic field simplification for charged particle motion is fully adequate if the particle trajectory remains in small region of space. However, in some case we can expect also large scale magnetic field that are close uniformity, for example, if a black hole is immersed in the field of closely positioned magnetar, or in the homogeneous part of the galactic magnetic field. Even in this case, charged particle motion in the combined gravomagnetic field has generally chaotic character.

In the case of external uniform magnetic fields we have demonstrated existence of four possible fates of inner regions of ionized Keplerian disks, corresponding to survival in the regime of oscillatory epicyclic motion, transformation of the originally thin disk to a thick toroidal structure, or destruction due to the fall into the black hole, or escape along magnetic field lines in the form of a wind, or jet, if matter escapes to infinity. In the last case, the magnetic Penrose process can lead to escape of extremely energetic charged particles.

Around Kerr black holes, charged particles from ionized Keplerian disks can be accelerated with efficiency of the magnetic Penrose process greater than $10^{10}$ so that protons can be accelerated to energy

- $\quad 10^{21} \mathrm{eV}$ around supermassive black hole with $M=10^{10} \mathrm{M}_{\odot}$ and $B=10^{4}$ Gauss

- $10^{15.6} \mathrm{eV}$ around supermassive black hole $\operatorname{SgrA}^{*}$ with $M=4 \cdot 10^{6} \mathrm{M}_{\odot}$ and $B=10$ Gauss

- $10^{19} \mathrm{eV}$ around supermassive black hole M87 with $M=7 \cdot 10^{9} \mathrm{M}_{\odot}$ and $B=100$ Gauss

Author Contributions: conceptualization, Z.S.; writing-draft preparation, review and editing, Z.S. and M.K.; formal analysis and analytic calculations, A.T. and M.K.; numerical calculations and visualization, M.K.; supervision, Z.S.;

Funding: This research received no external funding.

Acknowledgments: The authors thanks the Research Centre of Theoretical Physics and Astrophysics of the Faculty of Philosophy and Science of Silesian University in Opava for support.

Conflicts of Interest: The authors declare no conflict of interest.

\section{References}

1. Carter, B. Black hole equilibrium states. In Black Holes (Les Astres Occlus); Dewitt, C., Dewitt, B.S., Eds.; Gordon and Breach Science Publishers: New York, NY, USA, 1973; pp. 57-214.

2. Frolov, V.P.; Shoom, A.A. Motion of charged particles near a weakly magnetized Schwarzschild black hole. Phys. Rev. D 2010, 82, 084034, doi:10.1103/PhysRevD.82.084034.

3. Kovář, J.; Kopáček, O.; Karas, V.; Stuchlík, Z. Off-equatorial orbits in strong gravitational fields near compact objects-II: Halo motion around magnetic compact stars and magnetized black holes. Class. Quantum Gravity 2010, 27, 135006, doi:10.1088/0264-9381/27/13/135006. 
4. Stuchlík, Z.; Kološ, M. Acceleration of the charged particles due to chaotic scattering in the combined black hole gravitational field and asymptotically uniform magnetic field. Eur. Phys. J. C 2016, 76, 32, doi:10.1140/epjc/s10052-015-3862-2.

5. Wald, R.M. Black hole in a uniform magnetic field. Phys. Rev. D 1974, 10, 1680-1685, doi:10.1103/PhysRevD.10.1680.

6. Kološ, M.; Stuchlík, Z.; Tursunov, A. Quasi-harmonic oscillatory motion of charged particles around a Schwarzschild black hole immersed in a uniform magnetic field. Class. Quantum Gravity 2015, 32, 165009, doi:10.1088/0264-9381/32/16/165009.

7. Tursunov, A.; Stuchlík, Z.; Kološ, M. Circular orbits and related quasi-harmonic oscillatory motion of charged particles around weakly magnetized rotating black holes. Phys. Rev. D 2016, 93, 084012, doi:10.1103/PhysRevD.93.084012.

8. Kološ, M.; Tursunov, A.; Stuchlík, Z. Possible signature of the magnetic fields related to quasi-periodic oscillations observed in microquasars. Eur. Phys. J. C 2017, 77, 860, doi:10.1140/epjc/s10052-017-5431-3.

9. Dadhich, N.; Tursunov, A.; Ahmedov, B.; Stuchlík, Z. The distinguishing signature of magnetic Penrose process. Mon. Not. R. Astron. Soc. Lett. 2018, 478, L89-L94, doi:10.1093/mnrasl/sly073.

10. Kovář, J.; Stuchlík, Z.; Karas, V. Off-equatorial orbits in strong gravitational fields near compact objects. Class. Quantum Gravity 2008, 25, 095011, doi:10.1088/0264-9381/25/9/095011.

11. Bakala, P.; Šrámková, E.; Stuchlík, Z.; Török, G. On magnetic-field-induced non-geodesic corrections to relativistic orbital and epicyclic frequencies. Class. Quantum Gravity 2010, 27, 045001, doi:10.1088/0264-9381/27/4/045001.

12. Bakala, P.; Urbanec, M.; Šrámková, E.; Stuchlík, Z.; Török, G. On magnetic-field-induced corrections to the orbital and epicyclic frequencies: Paper II. Slowly rotating magnetized neutron stars. Class. Quantum Gravity 2012, 29, 065012, doi:10.1088/0264-9381/29/6/065012.

13. Bardeen, J.M.; Petterson, J.A. The Lense-Thirring Effect and Accretion Disks around Kerr Black Holes. Astrophys. J. 1975, 195, L65, doi:10.1086/181711.

14. Tursunov, A.A.; Stuchlík, Z.; Kološ, M.; Dadhich, N.; Ahmedov, B. Supermassive Black Hole as a source of Ultra-High-Energy Cosmic Rays. in preparation 2019.

15. Petterson, J.A. Magnetic field of a current loop around a Schwarzschild black hole. Phys. Rev. D 1974, 10, 3166-3170, doi:10.1103/PhysRevD.10.3166.

16. Nakamura, M.; Asada, K.; Hada, K.; Pu, H.Y.; Noble, S.; Tseng, C.; Toma, K.; Kino, M.; Nagai, H.; Takahashi, K.; et al. Parabolic Jets from the Spinning Black Hole in M87. Astrophys. J. 2018, 868, 146, doi:10.3847/1538-4357/aaeb2d.

17. Gammie, C.F.; McKinney, J.C.; Tóth, G. HARM: A Numerical Scheme for General Relativistic Magnetohydrodynamics. Astrophys. J. 2003, 589, 444-457, doi:10.1086/374594.

18. Janiuk, A.; Sapountzis, K.; Mortier, J.; Janiuk, I. Numerical simulations of black hole accretion flows. arXiv 2018, arXiv:1805.11305,

19. Bisnovatyi-Kogan, G.S.; Ruzmaikin, A.A. The Accretion of Matter by a Collapsing Star in the Presence of a Magnetic Field. Astrophys. Space Sci. 1974, 28, 45-59, doi:10.1007/BF00642237.

20. Bisnovatyi-Kogan, G.S.; Ruzmaikin, A.A. The Accretion of Matter by a Collapsing Star in the Presence of a Magnetic Field. II: Self-consistent Stationary Picture. Astrophys. Space Sci. 1976, 42, 401-424, doi:10.1007/BF01225967.

21. Bisnovatyi-Kogan, G.S. Accretion into Black Hole, and Formation of Magnetically Arrested Accretion Disks. Universe 2019, 5, 146, doi:10.3390/universe5060146.

22. Kopáček, O.; Karas, V.; Kovář, J.; Stuchlík, Z. Application of a symplectic integrator in a non-integrable relativistic system. In RAGtime 10-13: Workshops on Black Holes and Neutron Stars; Stuchlík, Z., Török, G., Pecháček, T., Eds.; Silesian University: Opava, Czech Republic, 2014; pp. 123-132.

23. Kološ, M.; Tursunov, A. Comparison of charged particle dynamics around compact object immersed into uniform or dipole magnetic field. In Proceedings of RAGtime 17-19: Workshops on Black Holes and Neutron Stars, 17-19/23-26 Oct., 1-5 Nov. 2015/2016/2017; Stuchlík, Z., Török, G., Karas, V., Eds.; Silesian University: Opava, Czech Republic, 2017; pp. 99-110, ISBN 978-80-7510-257-7.

24. Pánis, R.; Kološ, M.; Stuchlík, Z. Determination of chaotic behaviour in time series generated by charged particle motion around magnetized Schwarzschild black holes. arXiv 2019, arXiv:1905.01186.

25. Bicak, J.; Janis, V. Magnetic fluxes across black holes. Mon. Not. R. Astron. Soc. 1985, 212, 899-915. 
26. Parthasarathy, S.; Wagh, S.M.; Dhurandhar, S.V.; Dadhich, N. High efficiency of the Penrose process of energy extraction from rotating black holes immersed in electromagnetic fields. Astrophys. J. 1986, 307, 38-46, doi:10.1086/164390.

27. Kopáček, O.; Karas, V. Near-horizon Structure of Escape Zones of Electrically Charged Particles around Weakly Magnetized Rotating Black Hole. Astrophys. J. 2018, 853, 53, doi:10.3847/1538-4357/aaa45f.

28. Wald, R.M. General Relativity; University of Chicago Press: Chicago, IL, USA, 1984.

29. Stuchlík, Z.; Kološ, M. String loops in the field of braneworld spherically symmetric black holes and naked singularities. J. Cosmol. Astropart. Phys. 2012, 10, 008, doi:10.1088/1475-7516/2012/10/008.

30. Stuchlík, Z.; Kološ, M. String loops oscillating in the field of Kerr black holes as a possible explanation of twin high-frequency quasiperiodic oscillations observed in microquasars. Phys. Rev. D 2014, 89, 065007, doi:10.1103/PhysRevD.89.065007.

31. Belloni, T.M.; Sanna, A.; Méndez, M. High-frequency quasi-periodic oscillations in black hole binaries. Mon. Not. R. Astron. Soc. 2012, 426, 1701-1709, doi:10.1111/j.1365-2966.2012.21634.x.

32. Abramowicz, M.A.; Kluźniak, W. A precise determination of black hole spin in GRO J1655-40. Astron. Astrophys. 2001, 374, L19-L20, doi:10.1051/0004-6361:20010791.

33. Török, G.; Abramowicz, M.A.; Kluźniak, W.; Stuchlík, Z. The orbital resonance model for twin peak kHz quasi periodic oscillations in microquasars. Astron. Astrophys. 2005, 436, 1-8, doi:10.1051/0004-6361:20047115.

34. McClintock, J.E.; Narayan, R.; Davis, S.W.; Gou, L.; Kulkarni, A.; Orosz, J.A.; Penna, R.F.; Remillard, R.A.; Steiner, J.F. Measuring the spins of accreting black holes. Class. Quantum Gravity 2011, 28, 114009, doi:10.1088/0264-9381/28/11/114009.

35. Stuchlík, Z.; Kotrlová, A.; Török, G. Multi-resonance orbital model of high-frequency quasi-periodic oscillations: Possible high-precision determination of black hole and neutron star spin. Astron. Astrophys. 2013, 552, A10, doi:10.1051/0004-6361/201219724.

36. Stuchlík, Z.; Kološ, M. Mass of intermediate black hole in the source M82 X-1 restricted by models of twin high-frequency quasi-periodic oscillations. Mon. Not. R. Astron. Soc. 2015, 451, 2575-2588, doi:10.1093/mnras/stv1120.

37. Stuchlík, Z.; Kološ, M. Models of quasi-periodic oscillations related to mass and spin of the GRO J1655-40 black hole. Astron. Astrophys. 2016, 586, A130, doi:10.1051/0004-6361/201526095.

38. Stella, L.; Vietri, M.; Morsink, S.M. Correlations in the Quasi-periodic Oscillation Frequencies of Low-Mass X-Ray Binaries and the Relativistic Precession Model. Astrophys. J. Lett. 1999, 524, L63-L66, doi:10.1086/312291.

39. Török, G.; Kotrlová, A.; Šrámková, E.; Stuchlík, Z. Confronting the models of 3:2 quasiperiodic oscillations with the rapid spin of the microquasar GRS 1915+105. Astron. Astrophys. 2011, 531, A59, doi:10.1051/0004-6361/201015549.

40. Penrose, R. Gravitational Collapse: The Role of General Relativity. Nuovo Cimento Riv. Ser. 1969, 1, 252.

41. Pierre Auger Collaboration. Observation of a large-scale anisotropy in the arrival directions of cosmic rays above $8 \times 10^{18} \mathrm{eV}$. Science 2017, 357, 1266-1270, doi:10.1126/science.aan4338.

42. Aab, A.; Abreu, P.; Aglietta, M.; Albuquerque, I.F.; Allekotte, I.; Almela, A.; Castillo, J.A.; Alvarez-Muñiz, J.; Anastasi, G.A.; Anchordoqui, L.; et al. An Indication of Anisotropy in Arrival Directions of Ultra-high-energy Cosmic Rays through Comparison to the Flux Pattern of Extragalactic Gamma-Ray Sources. Astrophys. J. 2018, 853, L29, doi:10.3847/2041-8213/aaa66d.

43. Greisen, K. End to the Cosmic-Ray Spectrum? Phys. Rev. Lett. 1966, 16, 748-750, doi:10.1103/PhysRevLett.16.748.

(c) 2019 by the authors. Licensee MDPI, Basel, Switzerland. This article is an open access article distributed under the terms and conditions of the Creative Commons Attribution (CC BY) license (http://creativecommons.org/licenses/by/4.0/). 